\title{
Trefftz Displacement Potential Function Method for Solving Elastic Half-Space Problems
}

\author{
Charles Chinwuba Ike ${ }^{1, *}$, Benjamin Okwudili Mama², Hyginus Nwankwo Onah², \\ Clifford Ugochukwu Nwoji \\ ${ }^{1}$ Department of Civil Engineering, Enugu State University of Science and Technology, Enugu State, Nigeria \\ ${ }^{2}$ Department of Civil Engineering, University of Nigeria, Nsukka, Enugu State, Nigeria
}

Received January 11, 2021; Revised March 19, 2021; Accepted April 18, 2021

\begin{abstract}
Cite This Paper in the following Citation Styles
(a): [1] Charles Chinwuba Ike, Benjamin Okwudili Mama, Hyginus Nwankwo Onah, Clifford Ugochukwu Nwoji, "Trefftz Displacement Potential Function Method for Solving Elastic Half-Space Problems," Civil Engineering and Architecture, Vol. 9, No. 3, pp. 559-583, 2021. DOI: 10.13189/cea.2021.090301.
\end{abstract}

(b): Charles Chinwuba Ike, Benjamin Okwudili Mama, Hyginus Nwankwo Onah, Clifford Ugochukwu Nwoji (2021). Trefftz Displacement Potential Function Method for Solving Elastic Half-Space Problems. Civil Engineering and Architecture, 9(3), 559-583. DOI: 10.13189/cea.2021.090301.

Copyright $\odot 2021$ by authors, all rights reserved. Authors agree that this article remains permanently open access under the terms of the Creative Commons Attribution License 4.0 International License

\begin{abstract}
The elastic half-space problem has been solved previously using Boussinesq, Papkovich, Love, and Green and Zerna, potential function methods. In this work, the Trefftz displacement potential function method is used to obtain the stress and displacement fields in an elastic half-space subjected to boundary loads. Point load and various distributed loads are considered. The problem is presented using displacement formulation as Navier-Lamé equations. It is proved that the Trefftz functions are solutions of the Navier-Lamé displacement equations. Strain fields are derived in terms of the Trefftz function using the strain-displacement relations. The stress fields are similarly derived. The Trefftz function for the case of a point load acting at the origin of the elastic half-space is derived using the double exponential Fourier transformation technique. Stress equilibrium boundary conditions are used to fully determine the Trefftz function. Stress and displacement fields for the point load are then determined. The solutions to stress and displacement fields for point load are then used as Green functions to obtain stress and displacement fields for uniformly distributed load over a finite line, circular area and rectangular area. It is found that the solutions obtained for the stress and displacement fields in the elastic half-space due to point and distributed loads are identical with previously obtained expressions, thus validating this work.
\end{abstract}

Keywords Trefftz Displacement Potential Function Method, Elastic Half-Space Problem, Stress Fields,
Displacement Fields, Boussinesq Problem

\section{Introduction}

\subsection{Background}

The elastic half-space problems are classical problems in the mathematical theory of three-dimensional (3D) elasticity. The central concerns of the elastic half-space problems are to determine the stress fields, and displacement fields due to applied loads on the loaded elastic media usually occupying the three-dimensional region of space defined in the 3D Cartesian coordinates system by $-\infty \leq x \leq \infty,-\infty \leq y \leq \infty, 0 \leq z \leq \infty, \quad[1-10]$. Elastic half-space problems are commonly found in elastic stress and elastic settlement analysis, advanced soil mechanics, foundation engineering, geotechnical engineering, and soil - structure interaction [11 - 23]. Specific types of the elastic half-space problems are the Boussinesq, Kelvin, Mindlin and Cerrutti problems. Boussinesq type problem of the elastic half-space considers load applied normally to the boundary surface of the elastic half-space and such that the boundary is free of shear stresses. In Cerrutti type problem of the elastic half-space the load is applied tangentially to the boundary of the elastic half-space [14]. Kelvin problem consists of 
finding stress and displacement fields in an elastic space $-\infty \leq x \leq \infty,-\infty \leq y \leq \infty,-\infty \leq z \leq \infty$ due to a point load, where the space region is considered linearly elastic and isotropic [15]. Kelvin problem is also the point load in an elastic (full) space problem. In Mindlin problem, an axisymmetric point load acts at the interior of a homogeneous, isotropic, elastic half-space. The objective of elastic half-space problems under known loads is to determine the stress and displacement fields.

The elastic half-space problems use the principles and methods of the mathematical theory of elasticity. The fundamental equations from which the governing equations are derived are the set of fifteen equations given by the three differential equations of equilibrium, the six equations of the generalised Hooke's stress-strain laws and the six kinematics equations relating strains and displacements [16 - 23]. The set of fifteen equations are required to be satisfied in addition to the boundary conditions of the problem imposed by the loads.

The elastic half-space medium can be considered homogeneous or non-homogeneous, isotropic, transversely isotropic (orthotropic) or anisotropic, linear elastic or non-linear elastic. The complexity of the analysis increases as the elastic half-space medium becomes non-homogeneous, anisotropic and non-linearly elastic. In this work, the elastic half-space medium is linearly elastic, isotropic and homogeneous, and this yields simplifications in the resulting governing equations.

Two fundamental techniques are commonly applied in the formulation of elasticity problems, namely displacement method and stress method. A third method called the mixed (hybrid) method is not commonly used. Displacement-based methods aim at reformulation of the set of fifteen equations to eliminate stress components and strain. The resulting equations have the three displacement components as the primary unknown variables [16 - 22]. Consequently, the system of fifteen equations reduces to a set of three coupled partial differential equations in terms of the three Cartesian components of the displacement. Stress-based methods of formulation of the elasticity problem involve the reformulation of the set of fifteen equations to eliminate strains and displacements and the equations reduce to a set of six equations in terms of the six Cauchy stress components in 3D problems. Mixed methods involve a reformulation of the set of fifteen equations such that the primary unknown variables are some components of stress, and some components of displacements.

Displacement-based methods of elasticity problems were derived and developed by Navier and Lamé. The equations are a system of three partial differential equations in terms of the three Cartesian components of the displacement field [16 - 22]. Stress-based methods of elasticity problems derived and developed by Airy, Beltrami, Michell, Morera, Maxwell and others are a system of six partial differential equations in terms of the six Cartesian stress components [16-22].
The development of the stress-based and displacement-based formulation methods for three-dimensional elasticity problems have resulted in the research on the development of solutions to the equations of the stress and displacement methods, which are called respectively stress and displacement functions [24 - 29]. Some stress functions for two- and three-dimensional elasticity problems are Airy, Morera, Maxwell, Beltrami-Michell and Love stress functions [29]. Some displacement functions include: Cerrutti, Boussinesq [24], Trefftz [25], Green and Zerna [26] and Boussinesq-Papkovich functions.

The advantage of displacement formulation of the elasticity problem of the half-space is that the problem simplifies from a system of fifteen equations to a system of three equations; and invariably to the determination of suitable displacement functions that would satisfy the boundary conditions of the problem.

Another merit of the displacement formulation that has been adopted in this work is that there are known catalogues of harmonic functions that could be chosen from in finding suitable displacement potential functions for given elastic half-space problem.

\subsection{Research Aim and Objectives}

The main aim of this work is to use the Trefftz displacement potential function method for finding stress and displacement fields in an elastic half-space $(-\infty \leq x \leq \infty ;-\infty \leq y \leq \infty ; 0 \leq z \leq \infty)$ due to point load and distributed loads applied on the boundary surface $(z=0)$ or the $x y$ Cartesian coordinate plane. The specific objectives include:

(i). to present the elastic half-space problem using displacement formulation as the Navier-Lamé equations.

(ii). to prove/show that the Trefftz displacement potential function $\Omega(x, y, z)$ are solutions of the Navier-Lamé displacement formulation of elastostatic problems of the half-space region $(-\infty \leq x \leq \infty ;-\infty \leq y \leq \infty ; 0 \leq z \leq \infty)$.

(iii). to derive expressions for strain and stress fields in terms of the Trefftz displacement potential functions.

(iv). to derive the Trefftz displacement potential function for a vertical point load $Q_{0}$ acting at the origin of the elastic half-space, and therefrom derive the expressions for stress and displacement fields in the elastic half-space due to a point load at the origin.

(v). to use the point load expressions for vertical stress field as Green functions and derive expressions for vertical stress field in the half-space due to distributed load over circular and rectangular loaded areas. 


\section{Theoretical Framework}

The governing equations of the theory of elasticity for the elastic half-space and half-plane problems consider simultaneously the requirements of kinematics relations, stress - strain laws and the differential equations of equilibrium [29-43].

\subsection{Strain-Displacement (Kinematic) Equations}

For infinitesimal strain or small displacement assumptions of the theory of elasticity, the strain-displacement (kinematic) equations are the following system of six equations:

$$
\begin{gathered}
\varepsilon_{x x}=\frac{\partial u}{\partial x} \\
\varepsilon_{y y}=\frac{\partial v}{\partial y} \\
\varepsilon_{z z}=\frac{\partial w}{\partial z} \\
\gamma_{x y}=\frac{\partial u}{\partial y}+\frac{\partial v}{\partial x} \\
\gamma_{y z}=\frac{\partial v}{\partial z}+\frac{\partial w}{\partial y} \\
\gamma_{z x}=\frac{\partial u}{\partial z}+\frac{\partial w}{\partial x}
\end{gathered}
$$

in which, $u, v, w$ are the displacement components in the $x$, $y$ and $z$ Cartesian coordinate directions, $\varepsilon_{x x}, \varepsilon_{y y}, \varepsilon_{z z}$ are the normal strains in the $x, y$ and $z$ Cartesian coordinate directions while $\gamma_{x y}, \gamma_{y z}$ and $\gamma_{z x}$ are the shear strains.

\subsection{Generalised Hooke's Stress - Strain Equations}

The generalised Hooke's stress-strain equations for three-dimensional (3D) isotropic, homogeneous linear elastic problems are given in terms of Lamé's constants by the set of six equations:

$$
\begin{gathered}
\sigma_{x x}=\lambda \varepsilon_{v}+2 G \varepsilon_{x x} \\
\sigma_{y y}=\lambda \varepsilon_{v}+2 G \varepsilon_{y y} \\
\sigma_{z z}=\lambda \varepsilon_{v}+2 G \varepsilon_{z z} \\
\tau_{x y}=\tau_{y x}=G \gamma_{x y} \\
\tau_{y z}=\tau_{z y}=G \gamma_{y z} \\
\tau_{z x}=\tau_{x z}=G \gamma_{x z}
\end{gathered}
$$

where $\lambda$ is the Lamé constant or Lamé coefficient, $G$ is the shear modulus of elasticity and $\varepsilon_{v}$ is the volumetric strain, given by:

$$
\varepsilon_{v}=\varepsilon_{x x}+\varepsilon_{y y}+\varepsilon_{z z}
$$

$\sigma_{x x}, \sigma_{y y}, \sigma_{z z}$ are normal stresses, $\tau_{x y}, \tau_{y z}, \tau_{z x}$ are shear stresses.

The shear modulus, $G$ is expressed in terms of the Young's modulus of elasticity, $E$ and the Poisson's ratio, $\mu$ as follows:

$$
G=\frac{E}{2(1+\mu)}
$$

The Lamé constant $\lambda$ is given in terms of the Young's modulus, $E$, and the Poisson's ratio, $\mu$, as follows:

$$
\lambda=\frac{\mu G}{(1+\mu)(1-2 \mu)}=\frac{2 \mu G}{1-2 \mu}
$$

\subsection{Differential Equations of Equilibrium}

The differential equations of equilibrium for the general case of elastodynamic problems are the system of equations:

$$
\begin{gathered}
\frac{\partial \sigma_{x x}}{\partial x}+\frac{\partial \tau_{y x}}{\partial y}+\frac{\partial \tau_{z x}}{\partial z}+f_{x}=\rho \ddot{u}=\rho \frac{\partial^{2} u}{\partial t^{2}} \\
\frac{\partial \tau_{x y}}{\partial x}+\frac{\partial \sigma_{y y}}{\partial y}+\frac{\partial \tau_{z y}}{\partial z}+f_{y}=\rho \ddot{v}=\rho \frac{\partial^{2} v}{\partial t^{2}} \\
\frac{\partial \tau_{x z}}{\partial x}+\frac{\partial \tau_{y z}}{\partial y}+\frac{\partial \sigma_{z z}}{\partial z}+f_{z}=\rho \ddot{w}=\rho \frac{\partial^{2} w}{\partial t^{2}} \\
\tau_{x y}=\tau_{y x} \\
\tau_{y z}=\tau_{z y} \\
\tau_{x z}=\tau_{z x}
\end{gathered}
$$

Where $f_{x}, f_{y}$ and $f_{z}$ are the $x, y$ and $z$ Cartesian components of the body force, $\rho$ is the mass density and the two dots over $u, v$ and $w$ denote second time derivatives.

When body forces are disregarded and the problem of elasticity considered is not a dynamic problem, but a static problem, the differential equations of equilibrium are simplified to be:

$$
\begin{aligned}
& \frac{\partial \sigma_{x x}}{\partial x}+\frac{\partial \tau_{y x}}{\partial y}+\frac{\partial \tau_{z x}}{\partial z}=0 \\
& \frac{\partial \tau_{x y}}{\partial x}+\frac{\partial \sigma_{y y}}{\partial y}+\frac{\partial \tau_{z y}}{\partial z}=0 \\
& \frac{\partial \tau_{x z}}{\partial x}+\frac{\partial \tau_{y z}}{\partial y}+\frac{\partial \sigma_{z z}}{\partial z}=0
\end{aligned}
$$




\section{Research Methodology}

\subsection{Navier-Lamé Displacement Formulation of 3D Elastostatic Problems}

Navier and Lamé working independently presented a displacement formulation of three-dimensional (3D) elastostatic problems which enormously simplified the system of fifteen independent differential equations governing the problem to a system of three coupled partial differential equations (PDEs) in terms of three unknown displacement components $u, v$ and $w$.

The Navier-Lamé displacement formulation of elastostatic problems for homogeneous, linear elastic, isotropic half-space is the following system of three coupled PDEs:

$$
\begin{aligned}
& \nabla^{2} u+\left(\frac{\lambda+G}{G}\right) \frac{\partial \varepsilon_{v}}{\partial x}=0 \\
& \nabla^{2} v+\left(\frac{\lambda+G}{G}\right) \frac{\partial \varepsilon_{v}}{\partial y}=0 \\
& \nabla^{2} w+\left(\frac{\lambda+G}{G}\right) \frac{\partial \varepsilon_{v}}{\partial z}=0
\end{aligned}
$$

in which $\nabla^{2}$ is the Laplacian operator, given by

$$
\nabla^{2}=\frac{\partial^{2}}{\partial x^{2}}+\frac{\partial^{2}}{\partial y^{2}}+\frac{\partial^{2}}{\partial z^{2}}
$$

Alternatively, the Navier-Lamé equations are:

$$
\begin{aligned}
& \nabla^{2} u+\left(\frac{\lambda+G}{G}\right) \frac{\partial}{\partial x}\left(\frac{\partial u}{\partial x}+\frac{\partial v}{\partial y}+\frac{\partial w}{\partial z}\right)=0 \\
& \nabla^{2} v+\left(\frac{\lambda+G}{G}\right) \frac{\partial}{\partial y}\left(\frac{\partial u}{\partial x}+\frac{\partial v}{\partial y}+\frac{\partial w}{\partial z}\right)=0 \\
& \nabla^{2} w+\left(\frac{\lambda+G}{G}\right) \frac{\partial}{\partial z}\left(\frac{\partial u}{\partial x}+\frac{\partial v}{\partial y}+\frac{\partial w}{\partial z}\right)=0
\end{aligned}
$$

\subsection{Displacement Potential Functions}

Scalar functions of the Cartesian coordinate space variables which are solutions of the Navier-Lamé displacement formulation equations and from which the displacement components $(u, v, w)$ could be derived are called displacement potential functions.

\subsection{Trefftz Displacement Potential Functions $\Omega(x, y, z)$}

Trefftz derived displacement potential function $\Omega(x, y, z)$ which satisfy the Navier-Lamé displacement formulation equations in terms of the displacement components as follows:

$$
\begin{gathered}
u(x, y, z)=\frac{\partial \Omega}{\partial x}+\left(\frac{\lambda+G}{G}\right) z \frac{\partial^{2} \Omega}{\partial x \partial z} \\
v(x, y, z)=\frac{\partial \Omega}{\partial y}+\left(\frac{\lambda+G}{G}\right) z \frac{\partial^{2} \Omega}{\partial y \partial z} \\
w(x, y, z)=-\left(\frac{\lambda+2 G}{G}\right) \frac{\partial \Omega}{\partial z}+\left(\frac{\lambda+G}{G}\right) z \frac{\partial^{2} \Omega}{\partial z^{2}}
\end{gathered}
$$

The condition for the Trefftz displacement function defined in Equations $(32-34)$ to be solutions to the Navier-Lamé equations is that the Trefftz function $\Omega(x, y, z)$ is biharmonic, and thus is satisfying the biharmonic equation in 3D Cartesian coordinate space variables. Thus,

$$
\nabla^{2} \nabla^{2} \Omega(x, y, z)=\left(\frac{\partial^{2}}{\partial x^{2}}+\frac{\partial^{2}}{\partial y^{2}}+\frac{\partial^{2}}{\partial z^{2}}\right)^{2} \Omega(x, y, z)=0(35)
$$

\subsection{Derivation of Strain Fields from the Trefftz Displacement Potential Function}

The strain fields are derived from the Trefftz functions by using the strain displacement relations in Equations (32 $-34)$. Thus,

$$
\begin{gathered}
\varepsilon_{x x}=\frac{\partial u}{\partial x}=\frac{\partial}{\partial x}\left(\frac{\partial \Omega}{\partial x}+\left(\frac{\lambda+G}{G}\right) z \frac{\partial^{2} \Omega}{\partial x \partial z}\right) \\
\varepsilon_{x x}=\frac{\partial^{2} \Omega}{\partial x^{2}}+\left(\frac{\lambda+G}{G}\right) z \frac{\partial^{3} \Omega}{\partial x^{2} \partial z}
\end{gathered}
$$

Similarly,

$$
\begin{gathered}
\varepsilon_{y y}=\frac{\partial v}{\partial y}=\frac{\partial}{\partial y}\left(\frac{\partial \Omega}{\partial y}+\left(\frac{\lambda+G}{G}\right) z \frac{\partial^{2} \Omega}{\partial y \partial z}\right) \\
\varepsilon_{y y}=\frac{\partial^{2} \Omega}{\partial y^{2}}+\left(\frac{\lambda+G}{G}\right) z \frac{\partial^{3} \Omega}{\partial y^{2} \partial z}
\end{gathered}
$$

Also,

$$
\begin{gathered}
\varepsilon_{z z}=\frac{\partial w}{\partial z}=\frac{\partial}{\partial z}\left(-\left(\frac{\lambda+G}{G}\right) \frac{\partial \Omega}{\partial z}+\left(\frac{\lambda+G}{G}\right) z \frac{\partial^{2} \Omega}{\partial Z^{2}}\right)(40) \\
\varepsilon_{z z}=-\left(\frac{\lambda+2 G}{G}\right) \frac{\partial^{2} \Omega}{\partial z^{2}}+\left(\frac{\lambda+G}{G}\right)\left(z \frac{\partial^{3} \Omega}{\partial z^{3}}+\frac{\partial^{2} \Omega}{\partial z^{2}}\right)(41) \\
\gamma_{x y}=\frac{\partial u}{\partial y}+\frac{\partial v}{\partial x}=\frac{\partial}{\partial y}\left(\frac{\partial \Omega}{\partial x}+\left(\frac{\lambda+G}{G}\right) z \frac{\partial^{2} \Omega}{\partial x \partial z}\right)+ \\
\frac{\partial}{\partial x}\left(\frac{\partial \Omega}{\partial y}+\left(\frac{\lambda+G}{G}\right) z \frac{\partial^{2} \Omega}{\partial y \partial z}\right)(42) \\
\gamma_{x y}=2\left(\frac{\partial^{2} \Omega}{\partial x \partial y}+\left(\frac{\lambda+G}{G}\right) z \frac{\partial^{3} \Omega}{\partial x \partial y \partial z}\right)
\end{gathered}
$$

provided 


$$
\begin{gathered}
\frac{\partial^{2} \Omega}{\partial x \partial y}=\frac{\partial^{2} \Omega}{\partial y \partial x} \\
\frac{\partial^{3} \Omega}{\partial y \partial x \partial z}=\frac{\partial^{3} \Omega}{\partial x \partial y \partial z} \\
\gamma_{y z}=\frac{\partial v}{\partial z}+\frac{\partial w}{\partial y}=\frac{\partial}{\partial z}\left(\frac{\partial \Omega}{\partial y}+\left(\frac{\lambda+G}{G}\right) z \frac{\partial^{2} \Omega}{\partial y \partial z}\right)+ \\
+\frac{\partial}{\partial y}\left(-\left(\frac{\lambda+2 G}{G}\right) \frac{\partial \Omega}{\partial z}+\left(\frac{\lambda+G}{G}\right) z \frac{\partial^{2} \Omega}{\partial z^{2}}\right) \\
\gamma_{y z}=2\left(\frac{\lambda+G}{G}\right) z \frac{\partial^{3} \Omega}{\partial y \partial z^{2}}
\end{gathered}
$$

provided

$$
\begin{gathered}
\frac{\partial^{2} \Omega}{\partial z \partial y}-\frac{\partial^{2} \Omega}{\partial y \partial z} \\
\gamma_{z x}=\frac{\partial w}{\partial x}+\frac{\partial u}{\partial z}=\frac{\partial}{\partial z}\left(\frac{\partial \Omega}{\partial x}+\left(\frac{\lambda+G}{G}\right) z \frac{\partial^{2} \Omega}{\partial x \partial z}\right)+ \\
+\frac{\partial}{\partial x}\left(-\left(\frac{\lambda+2 G}{G}\right) \frac{\partial \Omega}{\partial z}+\left(\frac{\lambda+G}{G}\right) z \frac{\partial^{2} \Omega}{\partial z^{2}}\right) \\
\gamma_{z x}=2\left(\frac{\lambda+G}{G}\right) z \frac{\partial^{3} \Omega}{\partial x \partial z^{2}}
\end{gathered}
$$

\subsection{Derivation of the Volumetric Strain in terms of the Trefftz Displacement Potential Function}

The volumetric strain is derived from the strain fields using Equation (13) to obtain:

$$
\begin{array}{r}
\varepsilon_{v}=\frac{\partial^{2} \Omega}{\partial x^{2}}+\left(\frac{\lambda+G}{G}\right) z \frac{\partial^{3} \Omega}{\partial x^{2} \partial z}+\frac{\partial^{2} \Omega}{\partial y^{2}}+ \\
+\left(\frac{\lambda+G}{G}\right) z \frac{\partial^{3} \Omega}{\partial y^{2} \partial z}-\left(\frac{\lambda+2 G}{G}\right) \frac{\partial^{2} \Omega}{\partial z^{2}}+ \\
+\left(\frac{\lambda+G}{G}\right) z \frac{\partial^{3} \Omega}{\partial z^{3}}+\left(\frac{\lambda+G}{G}\right) \frac{\partial^{2} \Omega}{\partial z^{2}} \\
\varepsilon_{v}=\left(\frac{\lambda+G}{G}\right) z \frac{\partial}{\partial z} \nabla^{2} \Omega+\nabla^{2} \Omega-2 \frac{\partial^{2} \Omega}{\partial z^{2}}
\end{array}
$$

But $\Omega$ is a harmonic function and we obtain

$$
\varepsilon_{v}=-2 \frac{\partial^{2} \Omega}{\partial Z^{2}}
$$

\subsection{Proof that the Trefftz Potential Function Satisfies the Navier-Lamé Displacement Formulation Equations}

By substitution of the Trefftz expressions for $u, v, w$ into the Navier-Lamé equations, we have:

$$
\nabla^{2}\left(\frac{\partial \Omega}{\partial x}+\left(\frac{\lambda+G}{G}\right) z \frac{\partial^{2} \Omega}{\partial x \partial z}\right)+\left(\frac{\lambda+G}{G}\right) \frac{\partial}{\partial x}\left(-2 \frac{\partial^{2} \Omega}{\partial Z^{2}}\right)=0
$$

Simplifying,

$$
\begin{gathered}
\nabla^{2} \frac{\partial \Omega}{\partial x}+\left(\frac{\lambda+G}{G}\right) \nabla^{2}\left(z \frac{\partial^{2} \Omega}{\partial x \partial z}\right)-2\left(\frac{\lambda+G}{G}\right) \frac{\partial}{\partial x}\left(\frac{\partial^{2} \Omega}{\partial Z^{2}}\right)=0 \\
\frac{\partial}{\partial x} \nabla^{2} \Omega+\left(\frac{\lambda+G}{G}\right) \nabla^{2}\left(z \frac{\partial^{2} \Omega}{\partial x \partial z}\right)-2\left(\frac{\lambda+G}{G}\right) \frac{\partial^{3} \Omega}{\partial x \partial Z^{2}}=0
\end{gathered}
$$

Similarly, for the expression for $v$,

$$
\nabla^{2}\left(\frac{\partial \Omega}{\partial y}+\left(\frac{\lambda+G}{G}\right) z \frac{\partial^{2} \Omega}{\partial y \partial z}\right)+\left(\frac{\lambda+G}{G}\right) \frac{\partial}{\partial y}\left(-2 \frac{\partial^{2} \Omega}{\partial z^{2}}\right)=0
$$

Simplifying,

$$
\frac{\partial}{\partial y} \nabla^{2} \Omega+\left(\frac{\lambda+G}{G}\right) \nabla^{2}\left(z \frac{\partial^{2} \Omega}{\partial y \partial z}\right)-2\left(\frac{\lambda+G}{G}\right) \frac{\partial^{3} \Omega}{\partial y \partial z^{2}}=0
$$

Also,

$$
\begin{array}{r}
\nabla^{2}\left(-\left(\frac{\lambda+2 G}{G}\right) \frac{\partial \Omega}{\partial z}+\left(\frac{\lambda+G}{G}\right) z \frac{\partial^{2} \Omega}{\partial z}\right)+ \\
+\left(\frac{\lambda+G}{G}\right) \frac{\partial}{\partial z}\left(-2 \frac{\partial^{2} \Omega}{\partial z^{2}}\right)=0
\end{array}
$$

Simplifying,

$$
\begin{gathered}
-\left(\frac{\lambda+2 G}{G}\right) \frac{\partial}{\partial z} \nabla^{2} \Omega-\frac{\partial}{\partial z} \nabla^{2} \Omega+\left(\frac{\lambda+G}{G}\right) \times \\
\times \nabla^{2}\left(z \frac{\partial^{2} \Omega}{\partial z^{2}}\right)-2\left(\frac{\lambda+G}{G}\right) \frac{\partial}{\partial z} \frac{\partial^{2} \Omega}{\partial z^{2}}=0(60) \\
\nabla^{2}\left(z \frac{\partial^{2} \Omega}{\partial x \partial z}\right)=\left(\frac{\partial^{2}}{\partial x^{2}}+\frac{\partial^{2}}{\partial y^{2}}+\frac{\partial^{2}}{\partial z^{2}}\right)\left(z \frac{\partial^{2} \Omega}{\partial x \partial z}\right)=0(61) \\
\nabla^{2}\left(z \frac{\partial^{2} \Omega}{\partial z^{2}}\right)=0 \\
\nabla^{2}\left(z \frac{\partial^{2} \Omega}{\partial y \partial z}\right)=0
\end{gathered}
$$

Equation (55), (58) and (60) simplify using Equations $(61-63)$ as:

$$
\begin{gathered}
\frac{\partial}{\partial x} \nabla^{2} \Omega-2\left(\frac{\lambda+G}{G}\right) \frac{\partial}{\partial x} \frac{\partial^{2} \Omega}{\partial Z^{2}}=0 \\
\frac{\partial}{\partial y} \nabla^{2} \Omega-2\left(\frac{\lambda+G}{G}\right) \frac{\partial}{\partial y} \frac{\partial^{2} \Omega}{\partial z^{2}}=0 \\
\frac{\partial}{\partial z} \nabla^{2} \Omega+2\left(\frac{\lambda+G}{G}\right) \frac{\partial}{\partial z} \frac{\partial^{2} \Omega}{\partial z^{2}}+\left(\frac{\lambda+G}{G}\right) \frac{\partial}{\partial z} \nabla^{2} \Omega=0(66)
\end{gathered}
$$

By differentiation with respect to $x$, Equation (64) becomes: 


$$
\begin{aligned}
& \frac{\partial}{\partial x}\left(\frac{\partial}{\partial x} \nabla^{2} \Omega-2\left(\frac{\lambda+G}{G}\right) \frac{\partial}{\partial x} \frac{\partial^{2} \Omega}{\partial Z^{2}}\right)=0 \\
& \frac{\partial^{2}}{\partial x^{2}} \nabla^{2} \Omega-2\left(\frac{\lambda+G}{G}\right) \frac{\partial^{2}}{\partial x^{2}} \frac{\partial^{2} \Omega}{\partial Z^{2}}=0
\end{aligned}
$$

By differentiation with respect to $y$, Equation (65) becomes

$$
\begin{aligned}
\frac{\partial}{\partial y}\left(\frac{\partial}{\partial y} \nabla^{2} \Omega-2\left(\frac{\lambda+G}{G}\right) \frac{\partial}{\partial y} \frac{\partial^{2} \Omega}{\partial Z^{2}}\right) & =0 \\
\frac{\partial^{2}}{\partial y^{2}} \nabla^{2} \Omega-2\left(\frac{\lambda+G}{G}\right) \frac{\partial^{2}}{\partial y^{2}} \frac{\partial^{2} \Omega}{\partial Z^{2}} & =0
\end{aligned}
$$

By differentiation with respect to $z$, Equation (66) becomes:

$$
\begin{gathered}
\frac{\partial}{\partial z}\left(\frac{\partial}{\partial z} \nabla^{2} \Omega+2\left(\frac{\lambda+G}{G}\right) \frac{\partial}{\partial z} \frac{\partial^{2} \Omega}{\partial z^{2}}+\right. \\
\left(\left(\frac{\lambda+G}{G}\right) \frac{\partial}{\partial z} \nabla^{2} \Omega\right)=0 \\
\frac{\partial^{2}}{\partial Z^{2}} \nabla^{2} \Omega+2\left(\frac{\lambda+G}{G}\right) \frac{\partial^{2}}{\partial Z^{2}} \frac{\partial^{2} \Omega}{\partial Z^{2}}+\left(\frac{\lambda+G}{G}\right) \frac{\partial^{2}}{\partial Z^{2}} \nabla^{2} \Omega=0
\end{gathered}
$$

Addition of Equations (68), (70) and (72) yield:

$$
\begin{gathered}
\frac{\partial^{2}}{\partial x^{2}} \nabla^{2} \Omega+\frac{\partial^{2}}{\partial y^{2}} \nabla^{2} \Omega+\frac{\partial^{2}}{\partial z^{2}} \nabla^{2} \Omega-2 \frac{\partial^{2}}{\partial Z^{2}} \nabla^{2} \Omega- \\
-2\left(\frac{\lambda+G}{G}\right)\left(\frac{\partial^{2}}{\partial x^{2}}+\frac{\partial^{2}}{\partial y^{2}}+\frac{\partial^{2}}{\partial z^{2}}\right) \times \\
\times \frac{\partial^{2} \Omega}{\partial z^{2}}-\left(\frac{\lambda+G}{G}\right) \frac{\partial^{2}}{\partial z^{2}} \nabla^{2} \Omega=0
\end{gathered}
$$

Simplifying,

$$
\begin{aligned}
& \left(\frac{\partial^{2}}{\partial x^{2}}+\frac{\partial^{2}}{\partial y^{2}}+\frac{\partial^{2}}{\partial z^{2}}\right) \nabla^{2} \Omega-2 \frac{\partial^{2}}{\partial z^{2}} \nabla^{2} \Omega- \\
& -2\left(\frac{\lambda+G}{G}\right) \nabla^{2} \frac{\partial^{2} \Omega}{\partial z^{2}}-\left(\frac{\lambda+G}{G}\right) \frac{\partial^{2}}{\partial z^{2}} \nabla^{2} \Omega=0
\end{aligned}
$$

Simplifying further,

$$
\nabla^{2} \nabla^{2} \Omega-2 \frac{\partial^{2}}{\partial Z^{2}} \nabla^{2} \Omega-3\left(\frac{\lambda+G}{G}\right) \frac{\partial^{2}}{\partial Z^{2}} \nabla^{2} \Omega=0
$$

The Trefftz displacementS potential function $\Omega(x, y, z)$ satisfies the three coupled Navier-Lamé displacement formulation equations if

$$
\nabla^{2} \Omega=0
$$

and

$$
\nabla^{2} \nabla^{2} \Omega=0
$$

and then Equation (75) would be identically satisfied. The Trefftz potential functions are thus proved to be solutions to the Navier-Lamé displacement equations of equilibrium.

\subsection{Derivation of the Stress Fields in terms of Trefftz Displacement Potential Function}

The stress fields are found in terms of the Trefftz function by using Equations $(7-12)$ as follows:

$$
\begin{gathered}
\sigma_{x x}=2 G\left(\frac{\partial^{2} \Omega}{\partial x^{2}}+\left(\frac{\lambda+G}{G}\right) z \frac{\partial^{3} \Omega}{\partial x^{2} \partial z}\right)+\lambda\left(-2 \frac{\partial^{2} \Omega}{\partial z^{2}}\right) \\
=2 G\left(\frac{\partial^{2} \Omega}{\partial x^{2}}+\left(\frac{\lambda+G}{G}\right) z \frac{\partial^{3} \Omega}{\partial x^{2} \partial z}-\frac{\lambda}{G} \frac{\partial^{2} \Omega}{\partial z^{2}}\right)(78) \\
\sigma_{y y}=2 G\left(\frac{\partial^{2} \Omega}{\partial y^{2}}+\left(\frac{\lambda+G}{G}\right) z \frac{\partial^{3} \Omega}{\partial y^{2} \partial z}\right)+\lambda\left(-2 \frac{\partial^{2} \Omega}{\partial z^{2}}\right) \\
=2 G\left(\frac{\partial^{2} \Omega}{\partial y^{2}}+\left(\frac{\lambda+G}{G}\right) z \frac{\partial^{3} \Omega}{\partial y^{2} \partial z}-\frac{\lambda}{G} \frac{\partial^{2} \Omega}{\partial z^{2}}\right)(79) \\
\sigma_{z z}=2 G\left(-\left(\frac{\lambda+2 G}{G}\right) \frac{\partial^{2} \Omega}{\partial z^{2}}+\left(\frac{\lambda+G}{G}\right) \times\right. \\
\left.\times\left(z \frac{\partial^{3} \Omega}{\partial z^{3}}+\frac{\partial^{2} \Omega}{\partial z^{2}}\right)\right)-2 \lambda \frac{\partial^{2} \Omega}{\partial z^{2}}=2(\lambda+G)\left(z \frac{\partial^{3} \Omega}{\partial z^{3}}-\frac{\partial^{2} \Omega}{\partial z^{2}}\right)(80) \\
\tau_{x y}=2 G\left(\frac{\partial^{2} \Omega}{\partial y \partial z}+\left(\frac{\lambda+G}{G}\right) z \frac{\partial^{3} \Omega}{\partial x \partial y \partial z}\right)(81) \\
\tau_{x z}=2(\lambda+G) z \frac{\partial^{3} \Omega}{\partial x \partial z^{2}}
\end{gathered}
$$

It is observed that on the $x y$ plane;

$$
\begin{gathered}
\tau_{x z}(x, y, z=0)=0 \\
\tau_{y z}(x, y, z=0)=0
\end{gathered}
$$

Thus, irrespective of the Trefftz displacement potential function, $\Omega(x, y, z)$ the shear stresses $\tau_{x z}(x, y, z=0)$ and $\tau_{z y}(x, y, z=0)$ vanish on the $x y$ coordinate plane. The Trefftz potential function defined in this work can only be applied to elastostatic problems in which the $x y$ coordinate plane given mathematically by the surface $z=0$ is free of shear stresses. The Trefftz potential function satisfies the shear stress free boundary conditions on the boundary surface of the elastic half-space. This observation about the shear stresses $\left(\tau_{x z}(x, y, z=0)=0 \quad\right.$ and $\left.\tau_{z y}(x, y, z=0)=0\right)$ on the $x y$ coordinate plate for any Trefftz potential function $\Omega(x, y, z)$ places a restriction on the type and class of $3 \mathrm{D}$ elasticity problems that could be solved using the Trefftz displacement potential function method presented herein. However, the class of 3D elastostatic problems involving elastic half-space subject to applied normal stresses only and where the boundary 
surface ( $x y$ coordinate plane) is not subject to shear forces (shear load or shear stresses) is an important class of problems in the theory of elasticity applied to advanced geotechnical problems and advanced soil/solid mechanics.

\section{Results}

\subsection{Suitable Trefftz Potential Function Using the Double Exponential Fourier Transform Method}

We desire to find a suitable Trefftz potential function $\Omega(x, y, z)$ which will satisfy the Laplace's equation in three dimensional (3D) Cartesian coordinates as well as the biharmonic equation in $3 \mathrm{D}$ space using the double exponential Fourier transform method.

By taking the double exponential Fourier transform of Equation (35), we obtain:

$$
\begin{aligned}
& \int_{-\infty}^{\infty} \int_{-\infty}^{\infty} \nabla^{2} \Omega(x, y, z) e^{i \beta_{1} x} e^{-i \beta_{2} y} d x d y=0 \\
& \int_{-\infty}^{\infty} \int_{-\infty}^{\infty} \nabla^{4} \Omega(x, y, z) e^{-i \beta_{1} x} e^{-i \beta_{2} y} d x d y=0
\end{aligned}
$$

In Equations (86) and (87), $\beta_{1}$ and $\beta_{2}$ are the exponential Fourier transform parameters.

Expansion yields:

$$
\begin{gathered}
\int_{-\infty}^{\infty} \int_{-\infty}^{\infty}\left(\frac{\partial^{2} \Omega}{\partial x^{2}}+\frac{\partial^{2} \Omega}{\partial y^{2}}+\frac{\partial^{2} \Omega}{\partial Z^{2}}\right) e^{-i \beta_{1} x} e^{-i \beta_{2} y} d x d y=0 \\
\int_{-\infty}^{\infty} \int_{-\infty}^{\infty}\left(\frac{\partial^{2}}{\partial x^{2}}+\frac{\partial^{2}}{\partial y^{2}}+\frac{\partial^{2}}{\partial Z^{2}}\right) \times \\
\left(\frac{\partial^{2} \Omega}{\partial x^{2}}+\frac{\partial^{2} \Omega}{\partial y^{2}}+\frac{\partial^{2} \Omega}{\partial Z^{2}}\right) e^{-i \beta_{1} x} e^{-i \beta_{2} y} d x d y=0
\end{gathered}
$$

By using the linearity property of the double Fourier exponential transformation, integration by parts and the Leibnitz formula, the transformation becomes:

$$
\begin{aligned}
& \frac{\partial^{2}}{\partial z} \int_{-\infty}^{\infty} \int_{-\infty}^{\infty} \Omega(x, y, z) e^{-i \beta_{1} x} e^{-i \beta_{2} y} d x d y- \\
& -\beta_{1}^{2} \int_{-\infty}^{\infty} \int_{-\infty}^{\infty} \Omega(x, y, z) e^{-i \beta_{1} x} e^{-i \beta_{2} y} d x d y- \\
& -\beta_{2}^{2} \int_{-\infty}^{\infty} \int_{-\infty}^{\infty} \Omega(x, y, z) e^{-i \beta_{1} x} e^{-i \beta_{2} y} d x d y=0
\end{aligned}
$$

Simplifying further,

$$
\frac{\partial^{2}}{\partial Z^{2}} \int_{-\infty}^{\infty} \int_{-\infty}^{\infty} \Omega(x, y, z) e^{-i \beta_{1} x} e^{-i \beta_{2} y} d x d y-
$$

$$
-\left(\beta_{1}^{2}+\beta_{2}^{2}\right) \int_{-\infty}^{\infty} \int_{-\infty}^{\infty} \Omega(x, y, z) e^{-i \beta_{1} x} e^{-i \beta_{2} y} d x d y=0
$$

Let

$$
\int_{-\infty}^{\infty} \int_{-\infty}^{\infty} \Omega(x, y, z) e^{-i \beta_{1} x} e^{-i \beta_{2} y} d x d y=\bar{\Omega}\left(\beta_{1}, \beta_{2}, z\right)(92)
$$

where $\bar{\Omega}\left(\beta_{1}, \beta_{2}, z\right)$ is the double exponential Fourier transform of the Trefftz function $\Omega(x, y, z)$ with respect to $x$ and $y$.

Then, Equation (91) can be expressed in terms of the Trefftz potential in the double exponential Fourier transform space as the homogeneous second order ordinary differential equation (ODE) given by:

$$
\frac{\partial^{2} \bar{\Omega}\left(\beta_{1}, \beta_{2}, z\right)}{\partial Z}-\left(\beta_{1}^{2}+\beta_{2}^{2}\right) \bar{\Omega}\left(\beta_{1}, \beta_{2}, z\right)=0 \text { (93) }
$$

The ODE in Equation (93) is solved using differential operator methods, trial function methods or any other method for solving ODEs. The general solution is obtained as:

$$
\begin{array}{r}
\bar{\Omega}\left(\beta_{1}, \beta_{2}, z\right)=c_{1} \exp \left(-\sqrt{\left(\beta_{1}^{2}+\beta_{2}^{2}\right) z}+\right. \\
+c_{2} \exp \left(\sqrt{\left(\beta_{1}^{2}+\beta_{2}^{2}\right) z}\right)
\end{array}
$$

In Equation (94), $c_{1}$ and $c_{2}$ are constants of integration.

For bounded solutions for stress and displacement fields, as $z \rightarrow \infty$, the Trefftz potential function $\bar{\Omega}\left(\beta_{1}, \beta_{2}, z\right)$ in the double exponential Fourier transform space is required to be finite and bounded as $z \rightarrow \infty$. Hence,

$$
c_{2}=0
$$

The general solution to the Trefftz potential function in the double exponential Fourier transforms space that meets the boundedness requirement is thus obtained as:

$$
\bar{\Omega}\left(\beta_{1}, \beta_{2}, z\right)=c_{1} \exp \left(-\sqrt{\left(\beta_{1}^{2}+\beta_{2}^{2}\right)} z\right)
$$

We observe that $\bar{\Omega}\left(\beta_{1}, \beta_{2}, z \rightarrow \infty\right) \rightarrow 0$.

By inversion, the Trefftz potential function is obtained in the physical domain variables as:

$$
\begin{gathered}
\Omega(x, y, z)=\frac{1}{(2 \pi)^{2}} \int_{-\infty}^{\infty} \int_{-\infty}^{\infty} \bar{\Omega}\left(\beta_{1}, \beta_{2}, z\right) e^{i \beta_{1} x} e^{i \beta_{2} y} d \beta_{1} d \beta_{2}(97) \\
\Omega(x, y, z)=\Omega=\frac{1}{(2 \pi)^{2}} \times \\
\times \int_{-\infty}^{\infty} \int_{-\infty}^{\infty} c_{1} \exp \left(-\sqrt{\left(\beta_{1}^{2}+\beta_{2}^{2}\right) z}\right) e^{i \beta_{1} x} e^{i \beta_{2} y} d \beta_{1} d \beta_{2}
\end{gathered}
$$

The Trefftz displacement potential function $\Omega(x, y, z)$ is obtained in terms of an unknown constant $c_{1}$ which can be found for particular problems of 3D elastostatic half-space problems using the boundary conditions. The boundary conditions would depend on the type of load 
applied i.e., whether the load is a point load or a distributed normal load applied over a known area of known configuration.

For the biharmonic condition, $\nabla^{4} \Omega=0$, we obtain by solving by exponential Fourier transformation, using integration by parts and the Leibnitz formula, and the method of trial functions:

$$
\begin{aligned}
& \bar{\Omega}\left(\beta_{1}, \beta_{2}, z\right)=c_{1} \exp \left(-\sqrt{\left(\beta_{1}^{2}+\beta_{2}^{2}\right)} z\right)+ \\
&+c_{2} \exp \sqrt{\left(\beta_{1}^{2}+\beta_{2}^{2}\right)} z+\bar{c}_{3} z \exp -\sqrt{\left(\beta_{1}^{2}+\beta_{2}^{2}\right)} z+ \\
&+\bar{c}_{4} z \exp \sqrt{\left(\beta_{1}^{2}+\beta_{2}^{2}\right)} z
\end{aligned}
$$

where $c_{1}, c_{2}, \bar{c}_{3}$ and $\bar{c}_{4}$ are integration constants.

$$
\text { Let } \begin{aligned}
\bar{C}_{3} & =-C_{3} \\
\bar{C}_{4} & =c_{4}
\end{aligned}
$$

Then,

$$
\begin{array}{r}
\bar{\Omega}\left(\beta_{1}, \beta_{2}, z\right)=\left(c_{1}-c_{3} z\right) \exp \left(-\sqrt{\left(\beta_{1}^{2}+\beta_{2}^{2}\right) z}\right)+ \\
+\left(c_{2}+c_{4} z\right) \exp \sqrt{\left(\beta_{1}^{2}+\beta_{2}^{2}\right) z}
\end{array}
$$

For bounded solutions

$$
\bar{\Omega}\left(\beta_{1}, \beta_{2}, z \rightarrow \infty\right) \rightarrow 0
$$

Then,

$$
c_{2}=c_{4}=0
$$

and,

$$
\bar{\Omega}\left(\beta_{1}, \beta_{2}, z\right)=\left(c_{1}-c_{3} z\right) \exp \left(-\sqrt{\left(\beta_{1}^{2}+\beta_{2}^{2}\right)} z\right)
$$

By inversion,

$$
\begin{gathered}
\Omega=\frac{1}{(2 \pi)^{2}} \int_{-\infty}^{\infty} \int_{-\infty}^{\infty}\left(c_{1}-c_{3} z\right) \times \\
\times \exp \left(-\sqrt{\left(\beta_{1}^{2}+\beta_{2}^{2}\right) z}\right) e^{i \beta x} e^{i \beta_{2} y} d \beta_{1} d \beta_{2} \\
\Omega=\frac{1}{(2 \pi)^{2}} \int_{-\infty}^{\infty} \int_{-\infty}^{\infty}\left(c_{1}-c_{3} z\right) e^{-\beta_{t} z} e^{i \beta_{1} x} e^{i \beta_{2} y} d \beta_{1} d \beta_{2}
\end{gathered}
$$

where

$$
\beta_{t}=\sqrt{\beta_{1}^{2}+\beta_{2}^{2}}
$$

The shear stress free boundary conditions $\tau_{x z}(x, y, z=0)$ and $\tau_{y z}(x, y, z=0)$ at point $x, y$ on the boundary surface $z=0$ are used together with the requirement of equilibrium of internal vertical stress and the applied load at the surface to find the two remaining integration constants in the expression for the Trefftz displacement potential function $\Omega$.

$$
\tau_{x z}=\frac{1}{(2 \pi)^{2}} \int_{-\infty}^{\infty} \int_{-\infty}^{\infty} c_{1} i \beta_{1} \beta_{t}^{2} e^{-\beta_{t} z} e^{i \beta_{1} x} e^{i \beta_{2} y} d \beta_{1} d \beta_{2}-
$$

$$
-\frac{1}{(2 \pi)^{2}} \int_{-\infty}^{\infty} \int_{-\infty}^{\infty} c_{3} i \beta_{1}\left(\beta_{t}^{2} z-2 \beta_{t}\right) e^{-\beta_{t} z} e^{i \beta_{1} x} e^{i \beta_{2} y} d \beta_{1} d \beta_{2}
$$

Similarly,

$$
\begin{gathered}
\tau_{y z}=\frac{1}{(2 \pi)^{2}} \int_{-\infty}^{\infty} \int_{-\infty}^{\infty} c_{1} i \beta_{2} \beta_{t}^{2} e^{-\beta_{t} z} e^{i \beta_{1} x} e^{i \beta_{2} y} d \beta_{1} d \beta_{2}- \\
-\frac{1}{(2 \pi)^{2}} \int_{-\infty}^{\infty} \int_{-\infty}^{\infty} c_{3} i \beta_{2}\left(\beta_{t}^{2} z-2 \beta_{t}\right) e^{-\beta_{t} z} e^{i \beta_{1} x} e^{i \beta_{2} y} d \beta_{1} d \beta_{2}(109) \\
\tau_{x z}(x, y, z=0)=\frac{1}{(2 \pi)^{2}} \int_{-\infty}^{\infty} \int_{-\infty}^{\infty} c_{1} i \beta_{1} \beta_{t}^{2} e^{i \beta_{1} x} e^{i \beta_{2} y} d \times \\
\times \beta_{1} d \beta_{2}-\frac{1}{(2 \pi)^{2}} \int_{-\infty}^{\infty} \int_{-\infty}^{\infty} c_{3} i \beta_{1}\left(-2 \beta_{t}\right) e^{i \beta_{1} x} e^{i \beta_{2} y} d \beta_{1} d \beta_{2}=0(110)
\end{gathered}
$$

Hence,

$$
\begin{gathered}
\beta_{t}^{2} c_{1}+2 \beta_{t} c_{3}=0 \\
c_{3}=-\frac{c_{1} \beta_{t}}{2}=-\frac{c_{1}}{2} \sqrt{\beta_{1}^{2}+\beta_{2}^{2}}
\end{gathered}
$$

The same result is obtained by applying Equation (84), $\tau_{x z}(x, y, z=0)=0$

Hence,

$$
\begin{array}{r}
\Omega=\frac{1}{(2 \pi)^{2}} \int_{-\infty}^{\infty} \int_{-\infty}^{\infty} c_{1}\left(1+\frac{\beta_{t} z}{2}\right) \times \\
\times e^{-\sqrt{\beta_{1}^{2}+\beta_{2}^{2}} z} e^{i \beta_{1} x} e^{i \beta_{2} y} d \beta_{1} d \beta_{2}
\end{array}
$$

\subsection{Trefftz Displacement Potential Function for Point Load of Magnitude $Q_{0}$ Acting at the Origin of the Elastic Half-Space}

The work considered a point load of magnitude $Q_{0}$ applied at the origin $(0,0,0)$ of the half-space on the $x y$ Cartesian coordinate plane (represented by $z=0$ ). The load is expressed using Dirac delta functions $\delta(x), \delta(y)$ as follows:

$$
P=Q_{0} \delta(x) \delta(y)
$$

The vertical stress field on the $x y$ Cartesian coordinate plane is given by:

$$
\begin{aligned}
\sigma_{z z}(x, y, z=0) & =2(\lambda+G)\left(0-\frac{\partial^{2} \Omega}{\partial Z^{2}}\right) \\
& =-2(\lambda+G) \frac{\partial^{2} \Omega}{\partial z^{2}}
\end{aligned}
$$

$$
\sigma_{z}(x, y, 0)=-2(\lambda+G) \frac{\partial^{2}}{\partial z^{2}} \frac{1}{(2 \pi)^{2}} \times
$$




$$
\begin{gathered}
\left.\times \int_{-\infty}^{\infty} \int_{-\infty}^{\infty}\left\{c_{1} \exp \left(-\sqrt{\left(\beta_{1}^{2}+\beta_{2}^{2}\right.}\right) z\right) e^{i \beta_{1} x} e^{i \beta_{2} y} d \beta_{1} d \beta_{2}\right\} \\
=\sigma_{z}(x, y, z=0)=-2(\lambda+G) \frac{1}{(2 \pi)^{2}} \times \\
\left.\times \int_{-\infty}^{\infty} \int_{-\infty}^{\infty}\left\{\left(\beta_{1}^{2}+\beta_{2}^{2}\right) c_{1} \exp \left(-\sqrt{\left(\beta_{1}^{2}+\beta_{2}^{2}\right.}\right) z\right) e^{i \beta_{1} x} e^{i \beta_{2} y} d \beta_{1} d \beta_{2}\right\} \\
\times \int_{-\infty}^{\infty} \int_{-\infty}^{\infty}\left(\beta_{1}^{2}+\beta_{2}^{2}\right) c_{1} e^{0} e^{i \beta_{1} x} e^{i \beta_{2} y} d \beta_{1} d \beta_{2}=\sigma_{z z}(x, y, z=0)= \\
=\frac{-2(\lambda+G)}{(2 \pi)^{2}} \int_{-\infty}^{\infty} \int_{-\infty}^{\infty}\left(\beta_{1}^{2}+\beta_{2}^{2}\right) c_{1} e^{i \beta_{1} x} e^{i \beta_{2} y} d \beta_{1} d \beta_{2}(117)
\end{gathered}
$$

The boundary condition obtained from the requirement of equilibrium of internal vertical stresses and the applied load is given by:

$$
\begin{gathered}
\sigma_{z z}(x, y, z=0)+Q_{0} \delta(x) \delta(y)=0 \\
-2(\lambda+G) \frac{\partial^{2} \Omega}{\partial z^{2}}=-Q_{0} \delta(x) \delta(y)
\end{gathered}
$$

Then, we obtain the boundary condition as:

$$
\begin{aligned}
& \frac{-2(\lambda+G)}{(2 \pi)^{2}} \int_{-\infty}^{\infty} \int_{-\infty}^{\infty}\left(\beta_{1}^{2}+\beta_{2}^{2}\right) c_{1} e^{i \beta_{1} x} e^{i \beta_{2} y} d \beta_{1} d \beta_{2} \\
& =\frac{-1}{(2 \pi)^{2}} \int_{-\infty}^{\infty} \int_{-\infty}^{\infty} Q_{0} \delta(x) \delta(y) e^{i \beta_{1} x} e^{i \beta_{2} y} d \beta_{1} d \beta_{2}
\end{aligned}
$$

Then,

$$
\begin{array}{r}
\frac{-2(\lambda+G)}{(2 \pi)^{2}}\left(\beta_{1}^{2}+\beta_{2}^{2}\right) c_{1}=\frac{-Q_{0} \delta(x) \delta(y)}{(2 \pi)^{2}} \\
c_{1}=\frac{Q_{0} \delta(x) \delta(y)}{\left(\beta_{1}^{2}+\beta_{2}^{2}\right) \cdot 2(\lambda+G)}
\end{array}
$$

Then,

$$
\begin{aligned}
\Omega(x, y, z)= & \frac{1}{(2 \pi)^{2}} \int_{-\infty}^{\infty} \int_{-\infty}^{\infty} \frac{Q_{0} \delta(x) \delta(y) \exp \left(-\sqrt{\left(\beta_{1}^{2}+\beta_{2}^{2}\right) z}\right)}{\left(\beta_{1}^{2}+\beta_{2}^{2}\right) 2(\lambda+G)} \times \\
& \times\left(1+\frac{\beta_{t} z}{2}\right) e^{i \beta_{1} x} e^{i \beta_{2} y} d \beta_{1} d \beta_{2} \\
\Omega(x, y, z)=\frac{Q_{0}}{(2 \pi)^{2} 2(\lambda+G)} \times & \\
= & \int_{-\infty}^{\infty} \int_{-\infty}^{\infty} \frac{\delta(x) \delta(y) \exp \left(-\sqrt{\left(\beta_{1}^{2}+\beta_{2}^{2}\right) z}\right)}{\left(\beta_{1}^{2}+\beta_{2}^{2}\right)} \times
\end{aligned}
$$

$$
\begin{gathered}
\times\left(1+\frac{\beta_{t} z}{2}\right) e^{i \beta_{1} x} e^{i \beta_{2} y} d \beta_{1} d \beta_{2} \\
\Omega(x, y, z)=\frac{Q_{0}}{(2 \pi)^{2} 2(\lambda+G)} \times \\
\times \int_{-\infty}^{\infty} \int_{-\infty}^{\infty} \frac{\exp \left(-\sqrt{\left(\beta_{1}^{2}+\beta_{2}^{2}\right)} z\right)}{\left(\beta_{1}^{2}+\beta_{2}^{2}\right)}\left(1+\frac{\beta_{t} z}{2}\right) d \beta_{1} d \beta_{2} \\
\Omega(x, y, z)=\frac{1}{2(\lambda+G)} \frac{Q_{0}}{2 \pi} \log _{e}(R+z) \\
=\frac{1}{2(\lambda+G)} \frac{Q_{0}}{2 \pi} \ln (R+z)
\end{gathered}
$$

where

$$
\begin{gathered}
R=\left(x^{2}+y^{2}+z^{2}\right)^{1 / 2} \\
\Omega=\frac{1}{2(\lambda+G)} \frac{Q_{0}}{2 \pi} \ln \left(z+\left(x^{2}+y^{2}+z^{2}\right)^{1 / 2}\right)
\end{gathered}
$$

In general,

$$
\Omega(x, y, z)=\frac{Q_{0} c_{1}}{2 \pi} \ln (z+R)
$$

where $c_{1}$ is a constant.

$$
c_{1}=\frac{1}{2(\lambda+G)}
$$

\subsubsection{Stress Fields}

The normal stress fields and shear stress fields are determined from Equations (78), (79), (80), (82 - 83) as follows:

$$
\begin{gathered}
\sigma_{z z}(x, y, z)=-\frac{3 \pi Q_{0}}{2 \pi} \frac{z^{3}}{R^{5}}=-\frac{Q_{0}}{z^{2}} K\left(\frac{r}{z}\right) \\
=-\frac{Q_{0}}{z}\left(1+\left(\frac{r}{z}\right)^{2}\right)^{-5 / 2} \frac{3}{2 \pi} \\
\tau_{y z}(x, y, z)=\frac{-3}{2 \pi} Q_{0} \frac{y z^{2}}{R^{5}} \\
\tau_{x z}(x, y, z)=\frac{-3}{2 \pi} Q_{0} \frac{x z^{2}}{R^{5}} \\
\sigma_{x x}(x, y, z)=\frac{-Q}{2 \pi R^{2}} \times \\
\left(\frac{3 x^{2} z}{R^{3}}-(1-2 \mu)\left(\frac{z}{R}-\frac{R}{R+z}+\frac{x^{2}(2 R+z)}{R(R+z)^{2}}\right)\right)(1 \\
\sigma_{y y}(x, y, z)=\frac{-Q}{2 \pi R^{2}} \times \\
\left(\frac{3 y^{2} z}{R^{3}}-(1-2 \mu)\left(\frac{x}{R}-\frac{R}{R+z}+\frac{y^{2}(2 R+z)}{R(R+z)^{2}}\right)\right)(1
\end{gathered}
$$




$$
\tau_{x y}=\frac{-Q x y}{2 \pi R^{2}}\left(\frac{3 z}{R^{3}}-\frac{(1-2 \mu)(2 R+z)}{R(R+z)}\right)
$$

Vertical stress influence coefficients $K(r / z)$ for values of $(r / z)$ have been calculated and presented in Table 1 for point load $Q_{0}$ at the origin of an elastic half-space.

\subsubsection{Displacement Fields}

The displacement fields are obtained by substitution of Equation (124) into Equations (32 - 34) as:

$$
\begin{aligned}
& u(x, y, z)=\frac{Q_{0}}{4 \pi G}\left(\frac{x z}{R^{3}}-\frac{(1-2 \mu) x}{R(R+z)}\right) \\
& v(x, y, z)=\frac{Q_{0}}{4 \pi G}\left(\frac{y z}{R^{3}}-\frac{(1-2 \mu) y}{R(R+z)}\right) \\
& w(x, y, z)=\frac{Q_{0}}{4 \pi G R}\left(\frac{z^{2}}{R^{2}}+2(1-\mu)\right) \\
& =\frac{Q_{0}}{\frac{4 \pi E R}{2(1+\mu)}}\left(\frac{z^{2}}{R^{2}}+2(1-\mu)\right) \\
& w(x, y, z)=\frac{Q_{0}}{2 \pi E R}\left(\frac{z^{2}}{R^{2}}+2(1-\mu)\right)= \\
& =\frac{2 Q_{0}\left(1-\mu^{2}\right)}{2 \pi E R}+\frac{Q_{0}(1+\mu)}{2 \pi E} \frac{Z^{2}}{R^{3}} \\
& w(x, y, z)=\frac{Q_{0}\left(1-\mu^{2}\right)}{\pi E R}+\frac{Q_{0}(1+\mu)}{2 \pi E} \frac{z^{2}}{R^{3}} \\
& w(x, y, z=0)=\frac{Q_{0}(1-\mu)}{2 \pi G\left(x^{2}+y^{2}\right)^{1 / 2}}=\frac{Q_{0}(1-\mu)}{2 \pi G r} \\
& =\frac{Q_{0}\left(1-\mu^{2}\right)}{\pi E r}
\end{aligned}
$$

\subsection{Solution for Vertical Fields due to Uniformly Distributed Load over Circular Foundation Areas}

The vertical stress field at an arbitrary point $A$ due to a uniformly distributed load of intensity $q_{0}$ acting over a circular foundation area of radius $r_{0}$ as shown in Figure 1 is determined using the vertical stress expression for point load determined as Equation (129) as Green function as follows:

$$
\sigma_{z}(x, y, z)=\int_{0}^{r_{0}} \int_{0}^{2 \pi} \frac{3 z^{3} q_{0} d x d y}{2 \pi R^{5}}
$$

By transformation to the polar coordinate system, we have:

$$
d x d y=|J| d r d \theta
$$

$|J|$ is the Jacobian of the transformation from the Cartesian to the polar coordinate system.

$$
|J|=\frac{\partial(x, y)}{\partial(r, \theta)}=\left|\begin{array}{ll}
\frac{\partial x}{\partial r} & \frac{\partial x}{\partial \theta} \\
\frac{\partial y}{\partial r} & \frac{\partial y}{\partial \theta}
\end{array}\right|
$$

The transformation equations are:

$$
\begin{gathered}
x=r \cos \theta \\
y=r \sin \theta \\
J=\left|\begin{array}{cc}
\cos \theta & -r \sin \theta \\
\sin \theta & r \cos \theta
\end{array}\right|=r
\end{gathered}
$$

By the cosine rule (law),

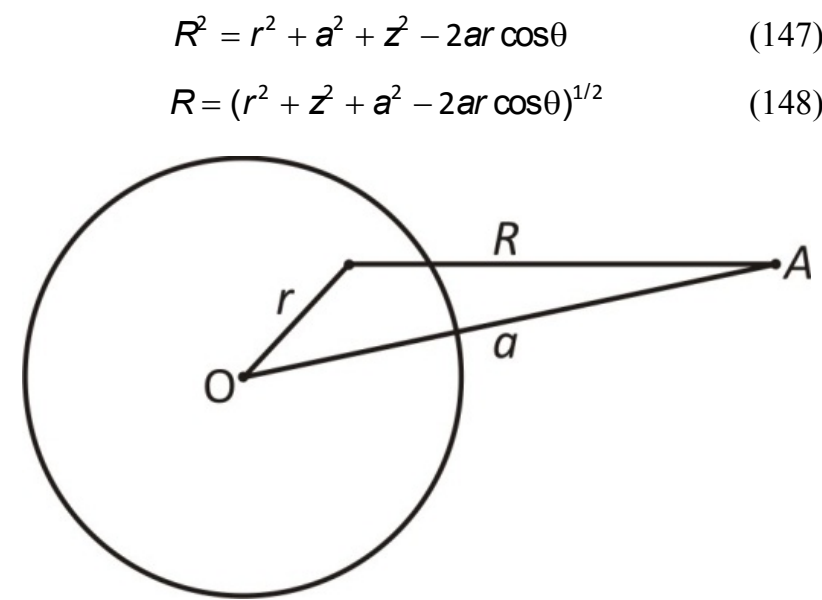

Figure 1. Circular foundation of radius $r_{0}$ under uniformly distributed load on elastic half-space

$$
\begin{gathered}
\sigma_{z z}(x, y, z)=\int_{0}^{r_{0}} \int_{0}^{2 \pi} \frac{3 z^{3} q_{0} r d r d \theta}{2 \pi\left(r^{2}+a^{2}+z^{2}-2 \operatorname{arcos} \theta\right)^{5 / 2}} \\
=\frac{3 z^{3} q_{0}}{2 \pi} \int_{0}^{r_{0}} \int_{0}^{2 \pi} \frac{r d r d \theta}{\left(r^{2}+z^{2}+a^{2}-2 a r \cos \theta\right)^{5 / 2}}
\end{gathered}
$$

Evaluating this double integration, we obtain the following expression, which is identical to the expressions obtained previously by Egorov and Screbrjanyi [30], [22] and Harr [31]:

$$
\begin{aligned}
\sigma_{Z z} & =q_{0}\left\{J-\frac{n}{\pi \sqrt{n^{2}+\left(1+t^{2}\right)}} \times\right. \\
& \left.\times\left[\frac{n^{2}-1+t^{2}}{n^{2}+(1-t)^{2}} E(k)+\frac{1-t}{1+t} \Pi_{0}(k, p)\right]\right\}
\end{aligned}
$$

Where $E(k)$ and $\prod_{0}(k, p)$ are complete elliptic integrals of the second and third kinds, respectively, with a modulus $k$, and parameter $p$ and

$$
t=\frac{r}{r_{0}}
$$




$$
\begin{gathered}
n=\frac{z}{r_{0}} \\
k^{2}=\frac{4 t}{n^{2}+(1+t)^{2}} \\
p=\frac{-4 t}{(1+t)^{2}} \\
J=1 \text { if } r<r_{0} \\
J=\frac{1}{2} \text { if } r=r_{0} \\
J=0 \text { if } r>r_{0}
\end{gathered}
$$

For the vertical stress field under the centre of the circular foundation, $r=0, t=0, k=0, p=0$, and Equation (141) becomes:

$$
\begin{aligned}
& \sigma_{Z z}=q_{0}\left\{1-\frac{n}{\pi \sqrt{\left(n^{2}+1\right)}}\left[\frac{n^{2}-1}{n^{2}+1} E(0)+\Pi_{0}(0,0)\right]\right\}(158) \\
& \sigma_{z z}=q_{0}\left\{1-\frac{1}{\left(\left(\frac{r_{0}}{z}\right)^{2}+1\right)^{3 / 2}}\right\}=q_{0}\left\{1-\left(\left(\frac{r_{0}}{z}\right)^{2}+1\right)^{-3 / 2}\right\}
\end{aligned}
$$

The vertical stress field at any point with coordinates $(r$, $z$ ) in the elastic half-space is:

$$
\sigma_{z z}(r, z)=q l(r, z)
$$

where

$$
I(r, z)=I\left(\frac{r}{r_{0}}, \frac{z}{r_{0}}\right)
$$

and are called vertical stress influence factors.

$I\left(\frac{r}{r_{0}}, \frac{z}{r_{0}}\right)$ is presented in Table 2 for various values of $r / r_{0}$ and $z / r_{0}$. Table 3 shows the vertical stress influence factors for vertical stress fields under the centre of uniformly loaded circular foundation areas on an elastic half-space.

\begin{tabular}{|c|c|c|c|c|c|c|c|}
\hline $\mathrm{r} / \mathrm{z}$ & $\mathrm{K}(\mathrm{r} / \mathrm{z})$ & $r / z$ & $\mathrm{~K}(\mathrm{r} / \mathrm{z})$ & $r / z$ & $\mathrm{~K}(\mathrm{r} / \mathrm{z})$ & $\mathrm{r} / \mathrm{z}$ & $\mathrm{K}(\mathrm{r} / \mathrm{z})$ \\
\hline 0.00 & 0.4775 & 0.45 & 0.3011 & 0.90 & 0.1083 & 1.35 & 0.0357 \\
\hline 0.01 & 0.4773 & 0.46 & 0.2955 & 0.91 & 0.1057 & 1.36 & 0.0348 \\
\hline 0.02 & 0.4770 & 0.47 & 0.2899 & 0.92 & 0.1031 & 1.37 & 0.0340 \\
\hline 0.03 & 0.4764 & 0.48 & 0.2843 & 0.93 & 0.1005 & 1.38 & 0.0332 \\
\hline 0.04 & 0.4756 & 0.49 & 0.2788 & 0.94 & 0.0981 & 1.39 & 0.0324 \\
\hline 0.05 & 0.4745 & 0.50 & 0.2733 & 0.95 & 0.0956 & 1.40 & 0.0317 \\
\hline 0.06 & 0.4732 & 0.51 & 0.2679 & 0.96 & 0.0933 & 1.41 & 0.0309 \\
\hline 0.07 & 0.4717 & 0.52 & 0.2625 & 0.97 & 0.0910 & 1.42 & 0.0302 \\
\hline 0.08 & 0.4699 & 0.53 & 0.2571 & 0.98 & 0.0887 & 1.43 & 0.0295 \\
\hline 0.09 & 0.4679 & 0.54 & 0.2518 & 0.99 & 0.0865 & 1.44 & 0.0288 \\
\hline 0.10 & 0.4657 & 0.55 & 0.2466 & 1.00 & 0.0844 & 1.45 & 0.0282 \\
\hline 0.11 & 0.4633 & 0.56 & 0.2414 & 1.01 & 0.0823 & 1.46 & 0.0275 \\
\hline 0.12 & 0.4607 & 0.57 & 0.2383 & 1.02 & 0.0803 & 1.47 & 0.0269 \\
\hline 0.13 & 0.4579 & 0.58 & 0.2313 & 1.03 & 0.0783 & 1.48 & 0.0263 \\
\hline 0.14 & 0.4548 & 0.59 & 0.2263 & 1.04 & 0.0764 & 1.49 & 0.0257 \\
\hline 0.15 & 0.4516 & 0.60 & 0.2214 & 1.05 & 0.0744 & 1.50 & 0.0251 \\
\hline 0.16 & 0.4482 & 0.61 & 0.2165 & 1.06 & 0.0727 & 1.51 & 0.0245 \\
\hline 0.17 & 0.4446 & 0.62 & 0.2117 & 1.07 & 0.0709 & 1.52 & 0.0240 \\
\hline 0.18 & 0.4409 & 0.63 & 0.2070 & 1.08 & 0.0691 & 1.53 & 0.0234 \\
\hline 0.19 & 0.4370 & 0.64 & 0.2024 & 1.09 & 0.0674 & 1.54 & 0.0229 \\
\hline 0.20 & 0.4329 & 0.65 & 0.1978 & 1.10 & 0.0658 & 1.55 & 0.0224 \\
\hline 0.21 & 0.4286 & 0.66 & 0.1934 & 1.11 & 0.0641 & 1.56 & 0.0219 \\
\hline 0.22 & 0.4242 & 0.67 & 0.1889 & 1.12 & 0.0626 & 1.57 & 0.0214 \\
\hline 0.23 & 0.4197 & 0.68 & 0.1846 & 1.13 & 0.0610 & 1.58 & 0.0209 \\
\hline 0.24 & 0.4151 & 0.69 & 0.1809 & 1.14 & 0.0595 & 1.59 & 0.0204 \\
\hline 0.25 & 0.4103 & 0.70 & 0.1762 & 1.15 & 0.0581 & 1.60 & 0.0200 \\
\hline
\end{tabular}

Table 1: Influence coefficients for vertical stresses in a homogeneous semi-infinite elastic soil due to surface point load

Point Load: The vertical normal stress $\sigma_{z z}$ at a point located at a depth $z$ below the surface of a homogeneous half-space at a horizontal distance, $r$ from the point of application of a point load, $Q_{0}$ is given by the equation:

$$
\sigma_{z}=\frac{Q_{0}}{z} K\left(\frac{r}{z}\right) \text { wherein, } K\left(\frac{r}{z}\right)=\frac{3}{2 \pi}\left[1+\left(\frac{r}{z}\right)^{2}\right]^{-5 / 2}
$$

Table 1. Boussinesq $=\mathrm{s}$ Vertical Stress Influence Factors (Coefficients) 
Table 1. Boussinesq=s Vertical Stress Influence Factors (Coefficients) (Continued)

\begin{tabular}{|c|c|c|c|c|c|c|c|}
\hline $\mathrm{r} / \mathrm{z}$ & $\mathrm{K}(\mathrm{r} / \mathrm{z})$ & $\mathrm{r} / \mathrm{z}$ & $\mathrm{K}(\mathrm{r} / \mathrm{z})$ & $\mathrm{r} / \mathrm{z}$ & $\mathrm{K}(\mathrm{r} / \mathrm{z})$ & $\mathrm{r} / \mathrm{z}$ & $\mathrm{K}(\mathrm{r} / \mathrm{z})$ \\
\hline 0.26 & 0.4054 & 0.71 & 0.1721 & 1.16 & 0.0567 & 1.61 & 0.0195 \\
\hline 0.27 & 0.4004 & 0.72 & 0.1681 & 1.17 & 0.0553 & 1.62 & 0.0191 \\
\hline 0.28 & 0.3954 & 0.73 & 0.1641 & 1.18 & 0.0539 & 1.63 & 0.0187 \\
\hline 0.29 & 0.3902 & 0.74 & 0.1603 & 1.19 & 0.0526 & 1.64 & 0.0183 \\
\hline 0.30 & 0.3849 & 0.75 & 0.1565 & 1.20 & 0.0513 & 1.65 & 0.0179 \\
\hline 0.31 & 0.3796 & 0.76 & 0.1527 & 1.21 & 0.0501 & 1.66 & 0.0175 \\
\hline 0.32 & 0.3742 & 0.77 & 0.1491 & 1.22 & 0.0489 & 1.67 & 0.0171 \\
\hline 0.33 & 0.3687 & 0.78 & 0.1455 & 1.23 & 0.0477 & 1.68 & 0.0167 \\
\hline 0.34 & 0.3632 & 0.79 & 0.1420 & 1.24 & 0.0466 & 1.69 & 0.0163 \\
\hline 0.35 & 0.3577 & 0.80 & 0.1386 & 1.25 & 0.0454 & 1.70 & 0.0160 \\
\hline 0.36 & 0.3521 & 0.81 & 0.1353 & 1.26 & 0.0443 & 1.71 & 0.0157 \\
\hline 0.37 & 0.3465 & 0.82 & 0.1320 & 1.27 & 0.0433 & 1.72 & 0.0153 \\
\hline 0.38 & 0.3408 & 0.83 & 0.1288 & 1.28 & 0.0422 & 1.73 & 0.0150 \\
\hline 0.39 & 0.3351 & 0.84 & 0.1257 & 1.29 & 0.0412 & 1.74 & 0.0147 \\
\hline 0.40 & 0.3294 & 0.85 & 0.1226 & 1.30 & 0.0402 & 1.75 & 0.0144 \\
\hline 0.41 & 0.3238 & 0.86 & 0.1196 & 1.31 & 0.0393 & 1.76 & 0.0141 \\
\hline 0.42 & 0.3181 & 0.87 & 0.1166 & 1.32 & 0.0384 & 1.77 & 0.0138 \\
\hline 0.43 & 0.3124 & 0.88 & 0.1138 & 1.33 & 0.0374 & 1.78 & 0.0135 \\
\hline 0.44 & 0.3068 & 0.89 & 0.1110 & 1.34 & 0.0365 & 1.79 & 0.0132 \\
\hline
\end{tabular}

Table 1. Boussinesq=s Vertical Stress Influence Factors (Coefficients) (Continued)

\begin{tabular}{|c|c|c|c|c|c|c|c|}
\hline $\mathrm{r} / \mathrm{z}$ & $\mathrm{K}(\mathrm{r} / \mathrm{z})$ & $\mathrm{r} / \mathrm{z}$ & $\mathrm{K}(\mathrm{r} / \mathrm{z})$ & $\mathrm{r} / \mathrm{z}$ & $\mathrm{K}(\mathrm{r} / \mathrm{z})$ & $\mathrm{r} / \mathrm{z}$ & $\mathrm{K}(\mathrm{r} / \mathrm{z})$ \\
\hline 1.80 & 0.0129 & 2.32 & 0.0047 & 2.84 & 0.0019 & 3.36 & 0.0009 \\
\hline 1.81 & 0.0126 & 2.33 & 0.0046 & 2.85 & 0.0019 & 3.37 & 0.0009 \\
\hline 1.82 & 0.0124 & 2.34 & 0.0045 & 2.86 & 0.0019 & 3.38 & 0.0009 \\
\hline 1.83 & 0.0121 & 2.35 & 0.0044 & 2.87 & 0.0019 & 3.39 & 0.0009 \\
\hline 1.84 & 0.0119 & 2.36 & 0.0043 & 2.88 & 0.0018 & 3.40 & 0.0009 \\
\hline 1.85 & 0.0116 & 2.37 & 0.0043 & 2.89 & 0.0018 & 3.41 & 0.0008 \\
\hline 1.86 & 0.0114 & 2.38 & 0.0042 & 2.90 & 0.0018 & 3.42 & 0.0008 \\
\hline 1.87 & 0.0112 & 2.39 & 0.0041 & 2.91 & 0.0017 & 3.43 & 0.0008 \\
\hline 1.88 & 0.0109 & 2.40 & 0.0040 & 2.92 & 0.0017 & 3.44 & 0.0008 \\
\hline 1.89 & 0.0107 & 2.41 & 0.0040 & 2.93 & 0.0017 & 3.45 & 0.0008 \\
\hline 1.90 & 0.0105 & 2.42 & 0.0039 & 2.94 & 0.0017 & 3.46 & 0.0008 \\
\hline 1.91 & 0.0103 & 2.43 & 0.0038 & 2.95 & 0.0016 & 3.47 & 0.0008 \\
\hline 1.92 & 0.0101 & 2.44 & 0.0038 & 2.96 & 0.0016 & 3.48 & 0.0008 \\
\hline 1.93 & 0.0099 & 2.45 & 0.0037 & 2.97 & 0.0016 & 3.49 & 0.0008 \\
\hline 1.94 & 0.0097 & 2.46 & 0.0036 & 2.98 & 0.0016 & 3.50 & 0.0007 \\
\hline 1.95 & 0.0095 & 2.47 & 0.0036 & 2.99 & 0.0015 & 3.55 & 0.0007 \\
\hline 1.96 & 0.0093 & 2.48 & 0.0035 & 3.00 & 0.0015 & 3.61 & 0.0007 \\
\hline 1.97 & 0.0091 & 2.49 & 0.0034 & 3.01 & 0.0015 & 3.62 & 0.0006 \\
\hline 1.98 & 0.0089 & 2.50 & 0.0034 & 3.02 & 0.0015 & 3.70 & 0.0006 \\
\hline 1.99 & 0.0087 & 2.51 & 0.0033 & 3.03 & 0.0014 & 3.74 & 0.0006 \\
\hline 2.00 & 0.0085 & 2.52 & 0.0033 & 3.04 & 0.0014 & 3.75 & 0.0005 \\
\hline
\end{tabular}


Table 1. Boussinesq $=\mathrm{s}$ Vertical Stress Influence Factors (Coefficients) (Continued)

\begin{tabular}{|c|c|c|c|c|c|c|c|}
\hline 2.01 & 0.0084 & 2.53 & 0.0032 & 3.05 & 0.0014 & 3.80 & 0.0005 \\
\hline 2.02 & 0.0082 & 2.54 & 0.0032 & 3.06 & 0.0014 & 3.90 & 0.0005 \\
\hline 2.03 & 0.0081 & 2.55 & 0.0031 & 3.07 & 0.0014 & 3.91 & 0.0004 \\
\hline 2.04 & 0.0079 & 2.56 & 0.0031 & 3.08 & 0.0013 & 4.00 & 0.0004 \\
\hline 2.05 & 0.0078 & 2.57 & 0.0030 & 3.09 & 0.0013 & 4.12 & 0.0004 \\
\hline 2.06 & 0.0076 & 2.58 & 0.0030 & 3.10 & 0.0013 & 4.13 & 0.0003 \\
\hline 2.07 & 0.0075 & 2.59 & 0.0029 & 3.11 & 0.0013 & 4.30 & 0.0003 \\
\hline 2.08 & 0.0073 & 2.60 & 0.0029 & 3.12 & 0.0013 & 4.43 & 0.0003 \\
\hline 2.09 & 0.0072 & 2.61 & 0.0028 & 3.13 & 0.0012 & 4.44 & 0.0002 \\
\hline 2.10 & 0.0070 & 2.62 & 0.0028 & 3.14 & 0.0012 & 4.70 & 0.0002 \\
\hline 2.11 & 0.0069 & 2.63 & 0.0027 & 3.15 & 0.0012 & 4.90 & 0.0002 \\
\hline 2.12 & 0.0068 & 2.64 & 0.0027 & 3.16 & 0.0012 & 4.91 & 0.0001 \\
\hline 2.13 & 0.0066 & 2.65 & 0.0026 & 3.17 & 0.0012 & 5.50 & 0.0001 \\
\hline 2.14 & 0.0065 & 2.66 & 0.0026 & 3.18 & 0.0012 & 6.15 & 0.0001 \\
\hline 2.15 & 0.0064 & 2.67 & 0.0025 & 3.19 & 0.0011 & & \\
\hline 2.16 & 0.0063 & 2.68 & 0.0025 & 3.20 & 0.0011 & & \\
\hline 2.17 & 0.0062 & 2.69 & 0.0025 & 3.21 & 0.0011 & & \\
\hline 2.18 & 0.0060 & 2.70 & 0.0024 & 3.22 & 0.0011 & & \\
\hline 2.19 & 0.0059 & 2.71 & 0.0024 & 3.23 & 0.0011 & & \\
\hline 2.20 & 0.0058 & 2.72 & 0.0023 & 3.24 & 0.0011 & & \\
\hline 2.21 & 0.0057 & 2.73 & 0.0023 & 3.25 & 0.0011 & & \\
\hline 2.22 & 0.0056 & 2.74 & 0.0023 & 3.26 & 0.0010 & & \\
\hline 2.23 & 0.0055 & 2.75 & 0.0022 & 3.27 & 0.0010 & & \\
\hline 2.24 & 0.0054 & 2.76 & 0.0022 & 3.28 & 0.0010 & & \\
\hline 2.25 & 0.0053 & 2.77 & 0.0022 & 3.29 & 0.0010 & & \\
\hline 2.26 & 0.0052 & 2.78 & 0.0021 & 3.30 & 0.0010 & & \\
\hline 2.27 & 0.0051 & 2.79 & 0.0021 & 3.31 & 0.0009 & & \\
\hline 2.28 & 0.0050 & 2.80 & 0.0021 & 3.32 & 0.0009 & & \\
\hline 2.29 & 0.0049 & 2.81 & 0.0020 & 3.33 & 0.0009 & & \\
\hline 2.30 & 0.0048 & 2.82 & 0.0020 & 3.34 & 0.0009 & & \\
\hline 2.31 & 0.0047 & 2.83 & 0.0020 & 3.35 & 0.0009 & & \\
\hline
\end{tabular}


Table 2. Vertical stress influence coefficients due to uniformly distributed load over a circular area of radius $r_{0}$ in semi-infinite linear elastic (elastic half space) soilVariation of $I$ with $z / r_{0}$ and $r / r_{0}$

\begin{tabular}{|c|c|c|c|c|c|c|c|c|c|}
\hline $\mathrm{z} / \mathrm{r}_{0}$ & $\mathbf{0}$ & 0.2 & 0.4 & 0.6 & 0.8 & 1 & 1.2 & 1.5 & 2 \\
\hline 0 & 1.0 & 1.0 & 1.0 & 1.0 & 1.0 & 0.5 & 0 & 0 & 0 \\
\hline 0.1 & 0.90050 & 0.89748 & 0.88679 & 0.86126 & 0.78797 & 0.43015 & 0.09645 & 0.02787 & 0.00856 \\
\hline 0.2 & 0.80388 & 0.79824 & 0.77884 & 0.73483 & 0.63014 & 0.38269 & 0.15433 & 0.05251 & 0.01680 \\
\hline 0.3 & 0.71265 & 0.70518 & 0.68316 & 0.62690 & 0.52081 & 0.34375 & 0.17964 & 0.07199 & 0.02440 \\
\hline 0.4 & 0.62861 & 0.62015 & 0.59241 & 0.53767 & 0.44329 & 0.31048 & 0.18709 & 0.08593 & 0.03118 \\
\hline 0.5 & 0.55279 & 0.54403 & 0.51622 & 0.46448 & 0.38390 & 0.28156 & 0.18556 & 0.09499 & 0.03701 \\
\hline 0.6 & 0.48550 & 0.47691 & 0.45078 & 0.40427 & 0.33676 & 0.25588 & 0.17952 & 0.10010 & \\
\hline 0.7 & 0.42654 & 0.41874 & 0.39491 & 0.35428 & 0.29833 & 0.21727 & 0.17124 & 0.10228 & 0.04558 \\
\hline 0.8 & 0.37531 & 0.36832 & 0.34729 & 0.31243 & 0.26581 & 0.21297 & 0.16206 & 0.10236 & \\
\hline 0.9 & 0.33104 & 0.32492 & 0.30669 & 0.27707 & 0.23832 & 0.19488 & 0.15253 & 0.10094 & \\
\hline 1 & 0.29289 & 0.28763 & 0.27005 & 0.24697 & 0.21468 & 0.17868 & 0.14329 & 0.09849 & 0.05185 \\
\hline 1.2 & 0.23178 & 0.22795 & 0.21662 & 0.19890 & 0.17626 & 0.15101 & 0.12570 & 0.09192 & 0.05260 \\
\hline 1.5 & 0.16795 & 0.16552 & 0.15877 & 0.14804 & 0.13436 & 0.11892 & 0.10296 & 0.08048 & 0.05116 \\
\hline 2 & 0.10557 & 0.10453 & 0.10140 & 0.09647 & 0.09011 & 0.08269 & 0.07471 & 0.06275 & 0.04496 \\
\hline 2.5 & 0.07152 & 0.07008 & 0.06947 & 0.06698 & 0.06373 & 0.05974 & 0.05555 & 0.04880 & 0.03787 \\
\hline 3 & 0.05132 & 0.05101 & 0.05022 & 0.04886 & 0.04707 & 0.04487 & 0.04241 & 0.03839 & 0.03150 \\
\hline 4 & 0.02986 & 0.02976 & 0.02907 & 0.02802 & 0.02832 & 0.02749 & 0.02651 & 0.02490 & 0.02193 \\
\hline 5 & 0.01942 & 0.01938 & & & & 0.01835 & & & 0.01573 \\
\hline 6 & 0.01361 & & & & & 0.01307 & & & 0.01168 \\
\hline 7 & 0.01005 & & & & & 0.00976 & & & 0.00894 \\
\hline 8 & 0.00772 & & & & & 0.00755 & & & 0.00703 \\
\hline 9 & 0.00612 & & & & & 0.00600 & & & 0.00566 \\
\hline 10 & & & & & & & & 0.00477 & 0.00465 \\
\hline
\end{tabular}

Table 2. Variation of $I$ with $z / r_{0}$ and $r / r_{0} \quad$ (continued)

\begin{tabular}{|c|c|c|c|c|c|c|c|c|c|}
\hline $\mathrm{z} / \mathrm{r}_{0} / \mathrm{r}_{0}$ & 3 & 4 & 5 & 6 & 7 & 8 & 10 & 12 & 14 \\
\hline 0 & 0 & 0 & 0 & 0 & 0 & 0 & 0 & 0 & 0 \\
\hline 0.1 & 0.00211 & 0.00084 & 0.00042 & & & & & & \\
\hline 0.2 & 0.00419 & 0.00167 & 0.00063 & 0.00048 & 0.00030 & 0.00020 & & & \\
\hline 0.3 & 0.00622 & 0.00250 & & & & & & & \\
\hline 0.5 & 0.01013 & 0.00407 & 0.00209 & 0.00118 & 0.00071 & 0.00053 & 0.00025 & 0.00014 & 0.00009 \\
\hline 1 & 0.01742 & 0.00761 & 0.00393 & 0.00236 & 0.00143 & 0.00097 & 0.00050 & 0.00029 & 0.00018 \\
\hline 1.2 & 0.01935 & 0.00871 & 0.00459 & 0.00269 & 0.00171 & 0.00115 & & & \\
\hline 1.5 & 0.02142 & 0.01013 & 0.00548 & 0.00325 & 0.00210 & 0.00141 & 0.00073 & 0.00043 & 0.00027 \\
\hline 2 & 0.02221 & 0.01160 & 0.00659 & 0.00399 & 0.00264 & 0.00180 & 0.00094 & 0.00056 & 0.00036 \\
\hline 2.5 & 0.02143 & 0.01221 & 0.00732 & 0.00463 & 0.00308 & 0.00214 & 0.00115 & 0.00068 & 0.00043 \\
\hline 3 & 0.01980 & 0.01220 & 0.00770 & 0.00505 & 0.00346 & 0.00242 & 0.00132 & 0.00079 & 0.00051 \\
\hline 4 & 0.01592 & 0.01109 & 0.00768 & 0.00536 & 0.00354 & 0.00282 & 0.00160 & 0.00099 & 0.00065 \\
\hline 5 & 0.01249 & 0.00949 & 0.00708 & 0.00527 & 0.00394 & 0.00298 & 0.00179 & 0.00113 & 0.00075 \\
\hline 6 & 0.00983 & 0.00795 & 0.00628 & 0.00492 & 0.00384 & 0.00299 & 0.00188 & 0.00124 & 0.00084 \\
\hline 7 & 0.00784 & 0.00661 & 0.00548 & 0.00443 & 0.00360 & 0.00291 & 0.00193 & 0.00130 & 0.00091 \\
\hline 8 & 0.00635 & 0.00554 & 0.00472 & 0.00398 & 0.00332 & 0.00276 & 0.00189 & 0.00134 & 0.00094 \\
\hline 9 & 0.00520 & 0.00466 & 0.00409 & 0.00353 & 0.00301 & 0.00256 & 0.00184 & 0.00133 & 0.00096 \\
\hline 10 & 0.00438 & 0.00397 & 0.00352 & 0.00326 & 0.00273 & 0.00241 & & & \\
\hline
\end{tabular}


4.3.1. Solutions for Vertical Displacement Fields Due to Uniformly Distributed Load on Circular Foundation Areas on Elastic Half-space

By using Equation (137) as the Green function, the vertical displacement field in the elastic half-space for uniform load on circular foundation is obtained as the double integral:

$$
\begin{aligned}
& w(x, y, z)=\int_{0}^{r_{0}} \int_{0}^{2 \pi}\left\{\frac{\left(1-\mu^{2}\right)}{\pi E R} q_{0}+\frac{(1+\mu)}{2 \pi E} \frac{z^{3}}{R^{3}} q_{0}\right\} d x d y(1 \\
& w=\int_{0}^{r_{0}} \int_{0}^{2 \pi} \frac{\left(1-\mu^{2}\right)}{\pi E} \frac{q_{0} d x d y}{\left(x^{2}+y^{2}+z^{2}\right)^{1 / 2}}+ \\
& +\int_{0}^{r_{0}} \int_{0}^{2 \pi} \frac{(1+\mu)}{2 \pi E} \frac{z^{3} q_{0} d x d y}{\left(x^{2}+y^{2}+z^{2}\right)^{3 / 2}} \\
& w=\left(\frac{1-\mu^{2}}{\pi E}\right) q_{0} \int_{0}^{r_{0}} \int_{0}^{2 \pi} \frac{d x d y}{\left(x^{2}+y^{2}+z^{2}\right)^{1 / 2}}+ \\
& +\left(\frac{1+\mu}{2 \pi E}\right) q_{0} z^{3} \int_{0}^{r_{0}} \int_{0}^{2 \pi} \frac{d x d y}{\left(x^{2}+y^{2}+z^{2}\right)^{3 / 2}} \\
& w(0,0, z)=\frac{2 q r_{0}\left(1-\mu^{2}\right)}{E}\left(\sqrt{\left(1+n^{2}\right)}-n\right)+ \\
& +\frac{2 q r_{0}\left(1-\mu^{2}\right)}{E} \frac{n}{2(1-\mu) \sqrt{\left(1+n^{2}\right)}} \\
& w(0,0, z)=\frac{2 q r_{0}\left(1-\mu^{2}\right)}{E}\left(1+\frac{n}{2(1-\mu) \sqrt{\left(1+n^{2}\right)}}\right)
\end{aligned}
$$

where

$$
\begin{gathered}
n^{2}=\frac{z^{2}}{r_{0}^{2}} \\
w(r, z=0)=\frac{2 q r_{0}\left(1-\mu^{2}\right)}{\pi E}[(1-t) K(k)+(1+t) E(k)](
\end{gathered}
$$

$K(k)$ and $E(k)$ are complete elliptic integrals of the first and second kind, respectively, with a modulus $k$, where

$$
k=\sqrt{\left(\frac{4 t}{(1+t)^{2}}\right)}
$$

And

$$
t^{2}=\frac{r^{2}}{r_{0}^{2}}
$$

For points under the centre of the circle, $r=0, t=0, k=0$, and the displacement becomes:

$$
w(r=0,0)=\frac{2 q r_{0}\left(1-\mu^{2}\right)}{\pi E}[K(0)+E(0)]
$$

$$
\begin{gathered}
w(r=0,0)=\frac{2 q r_{0}\left(1-\mu^{2}\right)}{\pi E}(0+\pi) \\
w(0,0)=\frac{2 q r_{0}\left(1-\mu^{2}\right)}{E}
\end{gathered}
$$

\subsection{Vertical Stress Fields in Elastic Half-Space due to Uniformly Distributed Line of Finite Length}

Continuous footings supporting external walls and/or partition walls may be idealized as uniformly loaded lines of finite extent as shown in Figure 2. The solution to vertical stress field for a point load is used as a Green function to determine the vertical stress for a uniform line load of finite length $L$ and intensity $p_{0}$ as follows:

$$
\sigma_{z}(x, y, z)=\int_{0}^{L} \frac{3 z^{2}}{2 \pi R^{5}} p_{0} d y
$$

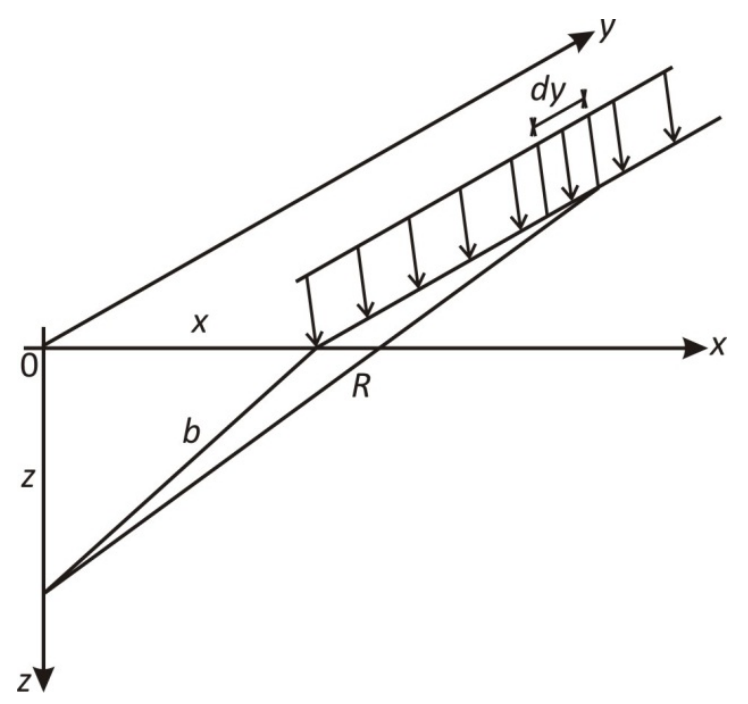

Figure 2. Uniformly loaded line of finite extent

$$
\sigma_{z z}(x, y, z)=\frac{3 z^{2} p_{0}}{2 \pi} \int_{0}^{L} \frac{d y}{\left(x^{2}+y^{2}+z^{2}\right)^{5 / 2}}
$$

The integral is evaluated by change of variables, where

$$
b^{2}=x^{2}+z
$$

and,

$$
y=b \tan \alpha
$$

then,

$$
\begin{gathered}
\sigma_{z}(x, y, z)=\frac{3 z^{3} p_{0}}{2 \pi} \int_{0}^{L} \frac{b \sec ^{2} \alpha d \alpha}{\left(b^{2}+b^{2} \tan ^{2} \alpha\right)^{5 / 2}} \\
=\frac{3 z^{3} p_{0}}{2 \pi b^{4}} \int_{0}^{L} \frac{d \alpha}{\sec ^{3} \alpha}=\frac{3 z^{3} p_{0}}{2 \pi b^{4}} \int_{0}^{L} \cos ^{3} \alpha d \alpha(178)
\end{gathered}
$$




$$
\begin{gathered}
\sigma_{z}(x, y, z)=\frac{3 z^{3} p_{0}}{2 \pi b^{4}}\left[\sin \alpha-\frac{\sin ^{3} \alpha}{3}\right]_{0}^{L} \\
=\frac{p_{0} z^{3}}{2 \pi}\left(\frac{L}{b^{2} \sqrt{\left(b^{2}+L^{2}\right)}}\left[\frac{2}{b^{2}}+\frac{1}{b^{2}+L^{2}}\right]\right) \\
\sigma_{z z}=\frac{p_{0}}{z} \frac{1}{2 \pi} \frac{L / z}{\left(1+\left(\frac{x}{z}\right)^{2}\right) \sqrt{\left(1+\left(\frac{x}{z}\right)^{2}+\left(\frac{L}{z}\right)^{2}\right)}} \times \\
\times\left[\frac{2}{1+\left(\frac{x}{z}\right)^{2}}+\frac{1}{\left(1+\left(\frac{x}{z}\right)^{2}+\left(\frac{L}{z}\right)^{2}\right)}\right] \\
\sigma_{z z}=\frac{p_{0}}{z} I\left(\frac{x}{z}, \frac{L}{z}\right)
\end{gathered}
$$

Let

$$
\begin{gathered}
\frac{x}{z}=m_{L} \\
\frac{L}{z}=n_{L} \\
\sigma_{z z}(x, y, z)=\frac{p_{0}}{z} \frac{1}{2 \pi}\left(\frac{n_{L}}{\left(m_{L}^{2}+1\right)}\right) \times \\
\frac{1}{\sqrt{\left(m_{L}^{2}+n_{L}^{2}+1\right)}}\left[\frac{2}{m_{L}^{2}+1}+\frac{1}{\left(m_{L}^{2}+n_{L}^{2}+1\right)}\right] \\
\sigma_{z}(x, y, z)=\frac{p_{0}}{z} I\left(m_{L}, n_{L}\right)
\end{gathered}
$$

where

$$
\begin{aligned}
I\left(m_{L}, n_{L}\right)= & \frac{1}{2 \pi}\left(\frac{n_{L}}{m_{L}^{2}+1}\right) \frac{1}{\sqrt{\left(m_{L}^{2}+n_{L}^{2}+1\right)}} \times \\
& \times\left[\frac{1}{\left(m_{L}^{2}+n_{L}^{2}+1\right)}+\frac{2}{\left(m_{L}^{2}+1\right)}\right]
\end{aligned}
$$

\subsection{Vertical Stress Fields due to Uniformly Distributed Load on a Rectangular Foundation Area $2 \mathrm{~L} \times 2 \mathrm{~B}$ on an Elastic Half-Space}

A rectangular area of dimensions $2 L \times 2 B$ which is subject to a distributed load of intensity $q$ was considered. By using the solution for a point load as Green function, the vertical stress distribution at point $(x, y, z)$ in an elastic half-space due to a distributed load of intensity $q$ on a rectangular area is given by:

$$
\sigma_{z}(x, y, z)=\int_{-L}^{L} \int_{-B}^{B} \frac{3 z^{3}}{2 \pi} \frac{q(\xi, \eta) d \xi d \eta}{\left((x-\xi)^{2}+(y-\eta)^{2}+z^{2}\right)^{5 / 2}}
$$

where $\xi, \eta$ are integration variables.

$$
\sigma_{z}(x, y, z)=\frac{3 z^{3}}{2 \pi} \int_{-L}^{L} \int_{-B}^{B} \frac{q(\xi, \eta) d \xi d \eta}{\left((x-\xi)^{2}+(y-\eta)^{2}+z^{2}\right)^{5 / 2}}
$$

For uniformly distributed loads, of intensity, $q_{0}$,

$$
q(\xi, \eta)=q_{0}
$$

and

$$
\begin{gathered}
\sigma_{z z}(x=0, y=0, z)= \\
2 \pi \int_{-L}^{L} \int_{-B}^{B} \frac{q_{0} d \xi d \eta}{\left((x-\xi)^{2}+(y-\eta)^{2}+z^{2}\right)^{5 / 2}} \\
\frac{3 q_{0} z^{3}}{2 \pi} \int_{-L}^{L} \int_{-B}^{B} \frac{d \xi d \eta}{\left((x-\xi)^{2}+(y-\eta)^{2}+z^{2}\right)^{5 / 2}} \\
\sigma_{z z}=q_{0} I_{0}(L, B, z)
\end{gathered}
$$

where

$$
I_{0}=\frac{3 z^{3}}{2 \pi} \int_{-L}^{L} \int_{-B}^{B} \frac{d \xi d \eta}{\left((x-\xi)^{2}+(y-\eta)^{2}+z^{2}\right)^{5 / 2}}
$$

$I_{0}(L, B, Z)$ is called the dimensionless vertical stress influence coefficient at the depth $z$ under the centre for a rectangular foundation $2 L \times 2 B$ carrying uniformly distributed load.

$$
\begin{aligned}
I_{0}(L, B, z)= & \frac{2}{\pi}\left\{\frac{L B z\left(L^{2}+B^{2}+2 Z^{2}\right)}{\left(L^{2}+Z^{2}\right)\left(B^{2}+Z^{2}\right) \sqrt{\left(L^{2}+B^{2}+Z^{2}\right)}}+\right. \\
& \left.+\sin ^{-1} \frac{L B}{\sqrt{\left(L^{2}+z^{2}\right)} \sqrt{\left(B^{2}+z^{2}\right)}}\right\}
\end{aligned}
$$

The $\sin ^{-1}$ function is in radian measure.

The vertical stress field at an arbitrary depth $z$ below the centre of a uniformly loaded rectangular foundation with sides $L \times B$ which rests on the surface of an elastic half-space is then:

$$
\begin{gathered}
\sigma_{\mathbb{Z C}}=\frac{1}{4} \sigma_{\mathbb{Z}}(x=0, y=0, z)=q_{0} I_{c}(L, B, z) \\
=\frac{1}{4} q_{0} I_{0}(L, B, z) \\
I_{c}(L, B, z)=\frac{1}{2 \pi}\left\{\frac{L B z\left(L^{2}+B^{2}+2 Z\right)}{\left(L^{2}+Z^{2}\right)\left(B^{2}+Z\right) \sqrt{\left(L^{2}+B^{2}+Z^{2}\right)}}+\right. \\
\left.+\sin ^{-1} \frac{L B}{\sqrt{\left(L^{2}+z^{2}\right) \sqrt{\left(B^{2}+z^{2}\right)}}}\right\}
\end{gathered}
$$

The results of the non-dimensional vertical stress influence coefficients are usually presented in terms of non-dimensional factors defined in terms of the length and width of the foundation as: 


$$
\begin{aligned}
& m_{1}=\frac{L}{B} \\
& n_{1}=\frac{z}{B}
\end{aligned}
$$

Then the vertical stress field at any depth $z$ below the centre of a uniformly loaded rectangular foundation area of dimensions $L \times B$ become

$$
\begin{aligned}
\sigma_{\mathbb{Z c}}\left(m_{1}, n_{1}\right)= & \frac{q_{0}}{2 \pi}\left\{\frac{m_{1} n_{1}}{\sqrt{\left(1+m_{1}^{2}+n_{1}^{2}\right)}} \frac{1+m_{1}^{2}+2 n_{1}^{2}}{\left(1+n_{1}^{2}\right)\left(m_{1}^{2}+n_{1}^{2}\right)}+\right. \\
& \left.+\sin ^{-1} \frac{m_{1}}{\sqrt{\left(m_{1}^{2}+n_{1}^{2}\right)\left(1+n_{1}^{2}\right)}}\right\}
\end{aligned}
$$

Thus,

$$
\sigma_{\mathbb{Z}}=q_{0} I_{c}\left(m_{1} n_{1}\right)
$$

where

$$
\begin{aligned}
I_{c}\left(m_{1} n_{1}\right)= & \frac{1}{2 \pi}\left\{\frac{m_{1} n_{1}\left(1+m_{1}^{2}+2 n_{1}^{2}\right)}{\sqrt{\left(1+m_{1}^{2}+n_{1}^{2}\right)\left(1+n_{1}^{2}\right)\left(m_{1}^{2}+n_{1}^{2}\right)}}+\right. \\
& \left.+\sin ^{-1} \frac{m_{1}}{\sqrt{\left(m_{1}^{2}+n_{1}^{2}\right)\left(1+n_{1}^{2}\right)}}\right\}
\end{aligned}
$$

Alternatively, for the dimensionless factors defined by

$$
\begin{aligned}
& m_{2}=\frac{L}{Z} \\
& n_{2}=\frac{B}{Z}
\end{aligned}
$$

The vertical stress influence coefficient becomes similar to results presented by Onah et al. [32] and is:

$$
\begin{gathered}
\sigma_{\mathbb{Z C}}=q_{0} I\left(m_{2}, n_{2}\right) \\
I\left(m_{2}, n_{2}\right)=\frac{1}{4 \pi}\left\{\frac{2 m_{2} n_{2}\left(m_{2}^{2}+n_{2}^{2}+1\right)^{1 / 2}}{\left(m_{2}^{2}+n_{2}^{2}+1+m_{2}^{2} n_{2}^{2}\right)} \frac{\left(m_{2}^{2}+n_{2}^{2}+2\right)}{\left(m_{2}^{2}+n_{2}^{2}+1\right)}+\right. \\
\left.+\tan ^{-1}\left(\frac{2 m_{2} n_{2}\left(1+m_{2}^{2}+n_{2}^{2}\right)^{1 / 2}}{\left(m_{2}^{2}+n_{2}^{2}-m_{2}^{2} n_{2}^{2}+1\right)}\right)\right\}
\end{gathered}
$$

The $\tan ^{-1}$ function is in radian measure.

When the dimensionless factors $m_{2}$ and $n_{2}$ are very small, the argument of $\tan ^{-1}$ becomes negative. The vertical stress influence coefficient for very small values of the dimensionless factors $m_{2}, n_{2}$ becomes:

$$
\begin{aligned}
I\left(m_{2}, n_{2}\right)= & \frac{1}{4 \pi}\left\{\frac{2 m_{2} n_{2} \sqrt{\left(m_{2}^{2}+n_{2}^{2}+1\right)}}{\left(m_{2}^{2}+n_{2}^{2}+m_{2}^{2} n_{2}^{2}+1\right)} \frac{\left(m_{2}^{2}+n_{2}^{2}+2\right)}{\left(m_{2}^{2}+n_{2}^{2}+1\right)}+\right. \\
& \left.+\tan ^{-1}\left(\pi-\left(\frac{2 m_{2} n_{2} \sqrt{\left(m_{2}^{2}+n_{2}^{2}+1\right)}}{m_{2}^{2}+n_{2}^{2}+1-m_{2}^{2} n_{2}^{2}}\right)\right)\right\}
\end{aligned}
$$

where $m_{2}=\frac{L}{z}, n_{2}=\frac{B}{z}$.

The non-dimensional factors $m_{2}$ and $n_{2}$ are interchangeable, and $I\left(m_{2}, n_{2}\right)$ is a symmetric function. Thus,

$$
I\left(m_{2}, n_{2}\right)=I\left(n_{2}, m_{2}\right)
$$

and $m_{2}$ and $n_{2}$ could have been defined interchangeably as $m_{2}=\frac{B}{z}, n_{2}=\frac{L}{z}$.

Values of the dimensionless vertical stress influence coefficients $I\left(m_{2}, n_{2}\right)$ for various values of the non-dimensional parameters $m_{2}, n_{2}$ have been calculated and shown in Table 4.

Table 3: Vertical normal stress field in a half-space beneath the centre of a uniformly loaded circular foundation area

The vertical normal stress at an arbitrary depth, $z$, beneath the centre of a circular foundation area with a radius $\boldsymbol{r}_{\mathbf{0}}$ carrying a uniformly distributed load of intensity $q$ per unit of area is $\sigma_{z}=q I$

wherein $I$ is the vertical stress influence coefficient.

$$
I=1-\left[1+\left(\frac{r_{0}}{z}\right)^{2}\right]^{-3 / 2}=I\left(\frac{r_{0}}{z}\right)
$$

The following table (Table 3 ) contains the values of $I$ for different values of $\left(r_{0} / z\right)$. 
Table 3. Vertical Influence Coefficient for Circular Foundation Areas Subject to Uniformly Distributed Loads

\begin{tabular}{|c|c|c|c|c|c|c|c|}
\hline$r_{0} / z$ & $I$ & $r_{0} / z$ & $I$ & $r_{0} / z$ & $I$ & $r_{0} / z$ & $I$ \\
\hline 0.00 & 0.00000 & 0.40 & 0.19959 & 0.80 & 0.52386 & 1.20 & 0.73763 \\
\hline 0.01 & 0.00015 & 0.41 & 0.20790 & 0.81 & 0.53079 & 1.21 & 0.74147 \\
\hline 0.02 & 0.00060 & 0.42 & 0.21627 & 0.82 & 0.53763 & 1.22 & 0.74525 \\
\hline 0.03 & 0.00135 & 0.43 & 0.22469 & 0.83 & 0.54439 & 1.23 & 0.74896 \\
\hline 0.04 & 0.00240 & 0.44 & 0.23315 & 0.84 & 0.55106 & 1.24 & 0.75262 \\
\hline 0.05 & 0.00374 & 0.45 & 0.24165 & 0.85 & 0.55766 & 1.25 & 0.75622 \\
\hline 0.06 & 0.00538 & 0.46 & 0.25017 & 0.86 & 0.56416 & 1.26 & 0.75976 \\
\hline 0.07 & 0.00731 & 0.47 & 0.25872 & 0.87 & 0.57058 & 1.27 & 0.76324 \\
\hline 0.08 & 0.00952 & 0.48 & 0.26729 & 0.88 & 0.57692 & 1.28 & 0.76666 \\
\hline 0.09 & 0.01203 & 0.49 & 0.27587 & 0.89 & 0.58317 & 1.29 & 0.77003 \\
\hline 0.10 & 0.01481 & 0.50 & 0.28446 & 0.90 & 0.58934 & 1.30 & 0.77334 \\
\hline 0.11 & 0.01788 & 0.51 & 0.29304 & 0.91 & 0.59542 & 1.31 & 0.77660 \\
\hline 0.12 & 0.02122 & 0.52 & 0.30162 & 0.92 & 0.60142 & 1.32 & 0.77981 \\
\hline 0.13 & 0.02483 & 0.53 & 0.31019 & 0.93 & 0.60734 & 1.33 & 0.78296 \\
\hline 0.14 & 0.02870 & 0.54 & 0.31875 & 0.94 & 0.61317 & 1.34 & 0.78606 \\
\hline 0.15 & 0.03283 & 0.55 & 0.32728 & 0.95 & 0.61892 & 1.35 & 0.78911 \\
\hline 0.16 & 0.03721 & 0.56 & 0.33579 & 0.96 & 0.62459 & 1.36 & 0.79211 \\
\hline 0.17 & 0.04184 & 0.57 & 0.34427 & 0.97 & 0.63018 & 1.37 & 0.79507 \\
\hline 0.18 & 0.04670 & 0.58 & 0.35272 & 0.98 & 0.63568 & 1.38 & 0.79797 \\
\hline 0.19 & 0.05181 & 0.59 & 0.36112 & 0.99 & 0.64110 & 1.39 & 0.80083 \\
\hline 0.20 & 0.05713 & 0.60 & 0.36949 & 1.00 & 0.64645 & 1.40 & 0.80364 \\
\hline 0.21 & 0.06268 & 0.61 & 0.37781 & 1.01 & 0.65171 & 1.41 & 0.80640 \\
\hline 0.22 & 0.06844 & 0.62 & 0.38609 & 1.02 & 0.65690 & 1.42 & 0.80912 \\
\hline 0.23 & 0.07441 & 0.63 & 0.39431 & 1.03 & 0.66200 & 1.43 & 0.81179 \\
\hline 0.24 & 0.08057 & 0.64 & 0.40247 & 1.04 & 0.66703 & 1.44 & 0.81442 \\
\hline 0.25 & 0.08692 & 0.65 & 0.41058 & 1.05 & 0.67198 & 1.45 & 0.81701 \\
\hline 0.26 & 0.09346 & 0.66 & 0.41863 & 1.06 & 0.67686 & 1.46 & 0.81955 \\
\hline 0.27 & 0.10017 & 0.67 & 0.42662 & 1.07 & 0.68166 & 1.47 & 0.82206 \\
\hline 0.28 & 0.10704 & 0.68 & 0.43454 & 1.08 & 0.68639 & 1.48 & 0.82452 \\
\hline 0.29 & 0.11408 & 0.69 & 0.44240 & 1.09 & 0.69104 & 1.49 & 0.82694 \\
\hline 0.30 & 0.12126 & 0.70 & 0.45018 & 1.10 & 0.69562 & 1.50 & 0.82932 \\
\hline 0.31 & 0.12859 & 0.71 & 0.45789 & 1.11 & 0.70013 & 1.51 & 0.83167 \\
\hline 0.32 & 0.13605 & 0.72 & 0.46553 & 1.12 & 0.70457 & 1.52 & 0.83397 \\
\hline 0.33 & 0.14363 & 0.73 & 0.47310 & 1.13 & 0.70894 & 1.53 & 0.83624 \\
\hline 0.34 & 0.15133 & 0.74 & 0.48059 & 1.14 & 0.71342 & 1.54 & 0.83847 \\
\hline 0.35 & 0.15915 & 0.75 & 0.48800 & 1.15 & 0.71747 & 1.55 & 0.84067 \\
\hline 0.36 & 0.16706 & 0.76 & 0.49533 & 1.16 & 0.72163 & 1.56 & 0.84283 \\
\hline 0.37 & 0.17507 & 0.77 & 0.50259 & 1.17 & 0.72573 & 1.57 & 0.84495 \\
\hline 0.38 & 0.18317 & 0.78 & 0.50976 & 1.18 & 0.72976 & 1.58 & 0.84704 \\
\hline 0.39 & 0.19134 & 0.79 & 0.51685 & 1.19 & 0.73373 & 1.59 & 0.84910 \\
\hline
\end{tabular}


Table 3. Vertical Influence Coefficient for Circular Foundation Areas Subject to Uniformly Distributed Loads (Continued)

\begin{tabular}{|c|c|c|c|c|c|c|c|}
\hline$r_{0} / z$ & $I$ & $r_{0} / z$ & $I$ & $r_{0} / z$ & $I$ & $r_{0} / z$ & $I$ \\
\hline 1.60 & 0.85112 & 1.90 & 0.89897 & 2.90 & 0.96536 & 7.00 & 0.99717 \\
\hline 1.61 & 0.85312 & 1.91 & 0.90021 & 2.95 & 0.96691 & 7.50 & 0.99769 \\
\hline 1.62 & 0.85507 & 1.92 & 0.90143 & & & & \\
\hline 1.63 & 0.85700 & 1.93 & 0.90263 & 3.00 & 0.96838 & 8.00 & 0.99809 \\
\hline 1.64 & 0.85890 & 1.94 & 0.90382 & 3.10 & 0.97106 & & \\
\hline 1.65 & 0.86077 & 1.95 & 0.90498 & 3.20 & 0.97346 & 9.00 & 0.99865 \\
\hline 1.66 & 0.86260 & 1.96 & 0.90613 & 3.30 & 0.97561 & & \\
\hline 1.67 & 0.86441 & 1.97 & 0.90726 & 3.40 & 0.97753 & 10.00 & 0.99901 \\
\hline 1.68 & 0.86619 & 1.98 & 0.90838 & 3.50 & 0.97927 & & \\
\hline 1.69 & 0.86794 & 1.99 & 0.90948 & 3.60 & 0.98083 & 12.00 & 0.99943 \\
\hline 1.70 & 0.86966 & 2.00 & 0.91056 & 3.70 & 0.98224 & 14.00 & 0.99964 \\
\hline 1.71 & 0.87136 & 2.02 & 0.91267 & 3.80 & 0.98352 & & \\
\hline 1.72 & 0.87302 & 2.04 & 0.91472 & 3.90 & 0.98468 & 16.00 & 0.99976 \\
\hline 1.73 & 0.87467 & 2.06 & 0.91672 & & & & \\
\hline 1.74 & 0.87628 & 2.08 & 0.91865 & 4.00 & 0.98573 & 18.00 & 0.99983 \\
\hline 1.75 & 0.87787 & 2.10 & 0.92053 & 4.20 & 0.98757 & & \\
\hline 1.76 & 0.87944 & 2.15 & 0.92499 & 4.40 & 0.98911 & 20.00 & 0.99988 \\
\hline 1.77 & 0.88098 & 2.20 & 0.92914 & 4.60 & 0.99041 & & \\
\hline 1.78 & 0.88250 & 2.25 & 0.93301 & 4.80 & 0.99152 & 25.00 & 0.99994 \\
\hline 1.79 & 0.88399 & $\begin{array}{l}2.30 \\
2.35 \\
\end{array}$ & $\begin{array}{l}0.93661 \\
0.93997 \\
\end{array}$ & & & 30.00 & 0.99996 \\
\hline 1.80 & 0.88546 & 2.40 & 0.94310 & 5.00 & 0.99246 & & \\
\hline 1.81 & 0.88691 & 2.45 & 0.94603 & 5.20 & 0.99327 & 40.00 & 0.99998 \\
\hline 1.82 & 0.88833 & 2.50 & 0.94877 & 5.40 & 0.99396 & & \\
\hline 1.83 & 0.88974 & 2.55 & 0.95134 & 5.60 & 0.99457 & 50.00 & 0.99999 \\
\hline 1.84 & 0.89112 & 2.60 & 0.95374 & 5.80 & 0.99510 & & \\
\hline 1.85 & 0.89248 & 2.65 & 0.95599 & & & 100.00 & 1.00000 \\
\hline 1.86 & 0.89382 & 2.70 & 0.95810 & 6.00 & 0.99556 & & \\
\hline 1.87 & 0.89514 & 2.75 & 0.96009 & 6.50 & 0.99648 & 4 & 1.00000 \\
\hline 1.88 & 0.89643 & 2.80 & 0.96195 & & & & \\
\hline 1.89 & 0.89771 & 2.85 & 0.96371 & & & & \\
\hline
\end{tabular}


Table 4. Dimensionless Influence Coefficients (Values or Factors) for Vertical Stresses in a Linear Elastic, Homogeneous Soil of Semi-infinite Extent Due to Uniformly Distributed Loads on rectangular foundation areas

\begin{tabular}{|c|c|c|c|c|c|c|c|c|c|c|c|c|}
\hline \multirow{2}{*}{$\mathrm{m}_{2}$} & \multicolumn{12}{|c|}{$\mathrm{n}_{2}$} \\
\hline & 0.1 & 0.2 & 0.3 & 0.4 & 0.5 & 0.6 & 0.7 & 0.8 & 0.9 & 1.0 & 1.2 & 1.4 \\
\hline 0.1 & 0.00470 & 0.00917 & 0.01323 & 0.01678 & 0.01978 & 0.02223 & 0.02420 & 0.02576 & 0.02698 & 0.02794 & 0.02926 & 0.03007 \\
\hline 0.2 & 0.00917 & 0.01790 & 0.02585 & 0.03280 & 0.03866 & 0.04348 & 0.04735 & 0.05042 & 0.05283 & 0.05471 & 0.05733 & 0.05894 \\
\hline 0.3 & 0.01323 & 0.02585 & 0.03735 & 0.04742 & 0.05593 & 0.06294 & 0.06858 & 0.07308 & 0.07661 & 0.07938 & 0.08323 & 0.08561 \\
\hline 0.4 & 0.01678 & 0.03280 & 0.04742 & 0.06024 & 0.07111 & 0.08009 & 0.08734 & 0.09314 & 0.09770 & 0.10129 & 0.10631 & 0.10941 \\
\hline 0.5 & 0.01978 & 0.03866 & 0.05593 & 0.07111 & 0.08403 & 0.09473 & 0.10340 & 0.11035 & 0.11584 & 0.12018 & 0.12626 & 0.13003 \\
\hline 0.6 & 0.02223 & 0.04348 & 0.06294 & 0.08009 & 0.09473 & 0.10688 & 0.11679 & 0.12474 & 0.13105 & 0.13605 & 0.14309 & 0.14749 \\
\hline 0.7 & 0.02420 & 0.04735 & 0.06858 & 0.08734 & 0.10340 & 0.11679 & 0.12772 & 0.13653 & 0.14356 & 0.14914 & 0.15703 & 0.16199 \\
\hline 0.8 & 0.02576 & 0.05042 & 0.07308 & 0.09314 & 0.11035 & 0.12474 & 0.13653 & 0.14607 & 0.15371 & 0.15976 & 0.16843 & 0.17389 \\
\hline 0.9 & 0.02698 & 0.05283 & 0.07661 & 0.09770 & 0.11584 & 0.13105 & 0.14356 & 0.15371 & 0.16185 & 0.16835 & 0.17766 & 0.18357 \\
\hline 1.0 & 0.02794 & 0.05471 & 0.07938 & 0.10129 & 0.12018 & 0.13605 & 0.14914 & 0.15978 & 0.16835 & 0.17522 & 0.18508 & 0.19139 \\
\hline 1.2 & 0.02926 & 0.05733 & 0.08323 & 0.10631 & 0.12626 & 0.14309 & 0.15703 & 0.16843 & 0.17766 & 0.18508 & 0.19584 & 0.20278 \\
\hline 1.4 & 0.03007 & 0.05894 & 0.08561 & 0.10941 & 0.13003 & 0.14749 & 0.16199 & 0.17389 & 0.18357 & 0.19139 & 0.20278 & 0.21020 \\
\hline 1.6 & 0.03058 & 0.05994 & 0.08709 & 0.11135 & 0.13241 & 0.15028 & 0.16515 & 0.17739 & 0.18737 & 0.19546 & 0.20731 & 0.21510 \\
\hline 1.8 & 0.03090 & 0.06058 & 0.08804 & 0.11260 & 0.13395 & 0.15207 & 0.16720 & 0.17967 & 0.18986 & 0.19814 & 0.21032 & 0.21836 \\
\hline 2.0 & 0.03111 & 0.06100 & 0.08867 & 0.11342 & 0.13496 & 0.15326 & 0.16856 & 0.18119 & 0.19152 & 0.19994 & 0.21235 & 0.22058 \\
\hline 2.5 & 0.03138 & 0.06155 & 0.08948 & 0.11450 & 0.13628 & 0.15483 & 0.17036 & 0.18321 & 0.19375 & 0.20236 & 0.21512 & 0.22364 \\
\hline 3.0 & 0.03150 & 0.06178 & 0.08982 & 0.11495 & 0.13684 & 0.15550 & 0.17113 & 0.18407 & 0.19470 & 0.20341 & 0.21633 & 0.22499 \\
\hline 4.0 & 0.03158 & 0.06194 & 0.09007 & 0.11527 & 0.13724 & 0.15598 & 0.17168 & 0.18469 & 0.19540 & 0.20417 & 0.21722 & 0.22600 \\
\hline 5.0 & 0.03160 & 0.06199 & 0.09014 & 0.11537 & 0.13737 & 0.15612 & 0.17185 & 0.18488 & 0.19561 & 0.20440 & 0.21749 & 0.22632 \\
\hline 6.0 & 0.03161 & 0.06201 & 0.09017 & 0.11541 & 0.13741 & 0.15617 & 0.17191 & 0.18496 & 0.19569 & 0.20449 & 0.21760 & 0.22644 \\
\hline 8.0 & 0.03162 & 0.06202 & 0.09018 & 0.11543 & 0.13744 & 0.15621 & 0.17195 & 0.18500 & 0.19574 & 0.20455 & 0.21767 & 0.22652 \\
\hline 10.0 & 0.03162 & 0.06202 & 0.09019 & 0.11544 & 0.13745 & 0.15622 & 0.17196 & 0.18502 & 0.19576 & 0.20457 & 0.21769 & 0.22654 \\
\hline$\infty$ & 0.03162 & 0.06202 & 0.09019 & 0.11544 & 0.13745 & 0.15623 & 0.17197 & 0.18502 & 0.19577 & 0.20458 & 0.21770 & 0.22656 \\
\hline
\end{tabular}


Table 4. Dimensionless Influence Coefficients (Values or Factors) for Vertical Stresses in a Linear Elastic, Homogeneous Soil of Semi-infinite Extent Due to Uniformly Distributed Loads on rectangular foundation areas (Continued)

\begin{tabular}{|c|c|c|c|c|c|c|c|c|c|c|c|}
\hline \multirow{2}{*}{$\mathrm{m}_{2}$} & \multicolumn{11}{|c|}{$\mathrm{n}_{2}$} \\
\hline & 1.6 & 1.8 & 2.0 & 2.5 & 3.0 & 4.0 & 5.0 & 6.0 & 8.0 & 10.0 & $\infty$ \\
\hline 0.1 & 0.03058 & 0.03090 & 0.03111 & 0.03138 & 0.03150 & 0.03158 & 0.03160 & 0.03161 & 0.03162 & 0.03162 & 0.03162 \\
\hline 0.2 & 0.05994 & 0.06058 & 0.06100 & 0.06155 & 0.06178 & 0.06194 & 0.06199 & 0.06201 & 0.06202 & 0.06202 & 0.06202 \\
\hline 0.3 & 0.08709 & 0.08804 & 0.08867 & 0.08948 & 0.08982 & 0.09007 & 0.09014 & 0.09017 & 0.09018 & 0.09019 & 0.09019 \\
\hline 0.4 & 0.11135 & 0.11260 & 0.11342 & 0.11450 & 0.11495 & 0.11527 & 0.11537 & 0.11541 & 0.11543 & 0.11544 & 0.11544 \\
\hline 0.5 & 0.13241 & 0.13395 & 0.13496 & 0.13628 & 0.13684 & 0.13724 & 0.13737 & 0.13741 & 0.13744 & 0.13745 & 0.13745 \\
\hline 0.6 & 0.15028 & 0.15207 & 0.15326 & 0.15483 & 0.15550 & 0.15598 & 0.15612 & 0.15617 & 0.15621 & 0.15622 & 0.15623 \\
\hline 0.7 & 0.16515 & 0.16720 & 0.16856 & 0.17036 & 0.17113 & 0.17168 & 0.17185 & 0.17191 & 0.17195 & 0.17196 & 0.17197 \\
\hline 0.8 & 0.17739 & 0.17967 & 0.18119 & 0.18321 & 0.18407 & 0.18469 & 0.18488 & 0.18496 & 0.18500 & 0.18502 & 0.18502 \\
\hline 0.9 & 0.18737 & 0.18986 & 0.19152 & 0.19375 & 0.19470 & 0.19540 & 0.19561 & 0.19569 & 0.19574 & 0.19576 & 0.19577 \\
\hline 1.0 & 0.19546 & 0.19814 & 0.19994 & 0.20236 & 0.20341 & 0.20417 & 0.20440 & 0.20449 & 0.20455 & 0.20457 & 0.20458 \\
\hline 1.2 & 0.20731 & 0.21032 & 0.21235 & 0.21512 & 0.21633 & 0.21722 & 0.21749 & 0.21760 & 0.21767 & 0.21769 & 0.21770 \\
\hline 1.4 & 0.21510 & 0.21836 & 0.22058 & 0.22364 & 0.22499 & 0.22600 & 0.22632 & 0.22644 & 0.22652 & 0.22654 & 0.22656 \\
\hline 1.6 & 0.22025 & 0.22372 & 0.22610 & 0.22940 & 0.23088 & 0.23200 & 0.23236 & 0.23249 & 0.23258 & 0.23261 & 0.23263 \\
\hline 1.8 & 0.22372 & 0.22736 & 0.22986 & 0.23334 & 0.23495 & 0.23617 & 0.23656 & 0.23671 & 0.23681 & 0.23684 & 0.23686 \\
\hline 2.0 & 0.22610 & 0.22986 & 0.23247 & 0.23614 & 0.23782 & 0.23912 & 0.23954 & 0.23970 & 0.23981 & 0.23985 & 0.23987 \\
\hline 2.5 & 0.22940 & 0.23334 & 0.23614 & 0.24010 & 0.24196 & 0.24344 & 0.24392 & 0.24412 & 0.24425 & 0.24429 & 0.24432 \\
\hline 3.0 & 0.23088 & 0.23495 & 0.23782 & 0.24196 & 0.24394 & 0.24554 & 0.24608 & 0.24630 & 0.24646 & 0.24650 & 0.24654 \\
\hline 4.0 & 0.23200 & 0.23617 & 0.23912 & 0.24344 & 0.24554 & 0.24729 & 0.24791 & 0.24817 & 0.24836 & 0.24842 & 0.24846 \\
\hline 5.0 & 0.23236 & 0.23656 & 0.23954 & 0.24392 & 0.24608 & 0.24791 & 0.24857 & 0.24885 & 0.24907 & 0.24914 & 0.24919 \\
\hline 6.0 & 0.23249 & 0.23671 & 0.23970 & 0.24412 & 0.24630 & 0.24817 & 0.24885 & 0.24916 & 0.24939 & 0.24946 & 0.24952 \\
\hline 8.0 & 0.23258 & 0.23681 & 0.23981 & 0.24425 & 0.24646 & 0.24836 & 0.24907 & 0.24939 & 0.24964 & 0.24973 & 0.24980 \\
\hline 10.0 & 0.23261 & 0.23684 & 0.23985 & 0.24429 & 0.24650 & 0.24842 & 0.24914 & 0.24946 & 0.24973 & 0.24981 & 0.24989 \\
\hline$\infty$ & 0.23263 & 0.23686 & 0.23987 & 0.24432 & 0.24654 & 0.24846 & 0.24919 & 0.24952 & 0.24980 & 0.24989 & 0.25000 \\
\hline
\end{tabular}

\section{Discussion}

The Trefftz displacement potential function method for determining the stresses and displacement fields in an elastic half-space $(-\infty \leq x \leq \infty,-\infty \leq y \leq \infty, 0 \leq z \leq \infty)$ due to loads on the boundary surface $(z=0)$ has been successfully presented in this work. The half-space material/medium considered was assumed linearly elastic, homogeneous and isotropic. The boundary loads considered in this study were point load $Q_{0}$ applied at the origin of the 3D elastic half-space, distributed line load of uniform intensity and of a finite length, uniformly distributed load over a circular area on the surface $(z=0)$ and uniformly distributed load over a given rectangular area on the surface.

The study adopted a displacement formulation. The set of fifteen equations that govern the elastic half-space problem were reformulated as a system of three coupled partial differential equations using the Navier-Lamé equations. It was shown that the Trefftz displacement potential function given by the system of PDEs in
Equations $(32$ - 34) are solutions of the Navier-Lamé displacement formulation of elastostatic problems of the half-space region provided the Trefftz function is a potential/harmonic function thus satisfying the 3D Laplace equation. The strain fields were derived from the Trefftz function presented as Equations (32 - 34) using the small displacement strain - displacement equations of the 3D elasticity theory. The strain fields were found in terms of the Trefftz function as Equations (37), (39), (41), (43), (47) and (50). The volumetric strain field was obtained in terms of the Trefftz potential function as Equation (53).

The generalized Hooke's stress - strain laws given in terms of Lamé's constants as Equations $(7-12)$ were used to obtain the stress fields in terms of the Trefftz displacement potential functions. The stress fields were obtained as Equations (78), (79), (80), (81), (82) and (83). It was observed from the stress field that shear stresses $\tau_{x z}$ and $\tau_{y z}$ vanish on the $x y$ plane, and thus the Trefftz displacement potential function $\Omega(x, y, z)$ satisfies the shear stress free condition on the $x y$ plane. This constrains the application of the Trefftz displacement potential 
function method adopted in this work to elastostatic problems in which the $x y$ coordinate plane is free of shear stresses.

A suitable Trefftz displacement potential function was found by applying the exponential Fourier transformation on the biharmonic partial differential equation and the Laplace equation. The exponential Fourier transformation which was given by Equation (88) was evaluated using the linearity property, integration by parts and the Leibnitz formula to yield a homogeneous second order ordinary differential equation (ODE) - Equation (93) - in the exponential Fourier transform space. The ODE was solved using differential (D) operator methods, trial function methods or other methods of solving ODE to obtain the general solution given by Equation (94) which contained two unknown integration constants. The requirements for stress and displacement fields to remain finite and bounded as $z \rightarrow \infty$ was used to require that the Trefftz function be finite and bounded as $z \rightarrow \infty$. Enforcement of the boundedness condition on the Trefftz potential function yielded the value of one of the unknown integration coefficients $c_{2}$, as zero, from Equation (95).

The bounded Trefftz displacement potential function was then obtained in the exponential Fourier transform space in terms of one unknown integration constant as Equation (96). The bounded Trefftz displacement potential function was obtained in the physical domain space variables by inversion as Equation (98). The bounded Trefftz displacement potential function given by Equation (98) is the general expression for $\Omega(x, y, z)$ and is not yet fully determined since there is still one unknown constant of integration in the expression for $\Omega(x, y, z)$.

The Trefftz displacement function that satisfies the biharmonic equation in 3D Cartesian coordinates was found by the exponential Fourier transformation technique as Equation (99) or (102). The requirement for bounded solutions for the Trefftz displacement function was used to obtain two integration constants as Equation (103). Thus, the bounded Trefftz displacement potential function that satisfies both the 3D Laplace equation as well as the biharmonic equation was obtained as Equation (104), and by inversion as Equation (105). Shear stress free boundary conditions on the $x y$ plane for $\tau_{x z}$ and $\tau_{y z}$ were used to obtain the relationship between the two remaining constants of integration as Equation (112). The Trefftz displacement potential function was then obtained in terms of one unknown integration constant as Equation (113). The requirement for equilibrium of internal vertical stress and the applied point load at the origin was used to obtain the integration constant $c_{1}$ as Equation (122). The Trefftz displacement potential function was thus consequently found for the point load at the origin of a semi-infinite half-space as Equation (123), and Equation (124) upon evaluation.

The full determination of the expression for the Trefftz displacement potential function is found to depend on the boundary conditions which are in turn dependent on the loading condition. For a point load applied at the origin of the elastic half-space, the boundary condition obtained from the requirement of vertical equilibrium of the internal vertical stresses and the stresses due to the applied point load was used to obtain the unknown integration constant $c_{1}$ as Equation (122).

The Trefftz displacement potential function for the case of point load applied at the origin was thus fully determined as Equations (124) or (126). The stress fields for the case of point load at the origin of the elastic half-space were then determined from the $\Omega(x, y, z)$ using Equations (78), (79), (80) and (82 - 83) as Equations (129134). The results agree with (Hankel transform method) solutions presented by Ike [21] using Hankel transform method. Vertical stress fields for point load at the origin are presented in terms of influence values in Table 1 . The displacement fields were similarly found by substitution of the Equation (124) into Equations $(32$ - 34). The displacement fields were obtained as Equations (135 - 137). The vertical displacement at the $x y$ plane was obtained as Equation (140). Three cases of distributed load, namely uniformly distributed load on a finite line, uniformly distributed load over a circular area and uniformly distributed load over a rectangular area were considered. For the case of uniformly distributed load over a circular area of known radius, the vertical stress field at any point in the elastic half-space was obtained by using the vertical stress field obtained for a point load at the origin as Green function. The vertical stress field for the case of uniformly distributed load over a circular area of radius $r_{0}$ was found to be given by the double integration problem over the circular foundation area given by Equation (141). The use of transformation method from Cartesian to cylindrical polar coordinates and the application of the cosine rule (formula) simplified the problem of the vertical stress field to Equation (149). The double integration problem in Equation (149) have been evaluated and solutions are also found by using Tables of integrals, and the vertical stress field at any point in an elastic half-space due to uniformly distributed load on circular foundations obtained as Equation (150). Equation (150) becomes a much simpler expression - Equation (159) - for points in the elastic half-space under the centre of the circular foundation. Equations (150) and (159) have been evaluated for different values of the ratios $r / r_{0}, z / r_{0}$ and the vertical stress expressions presented in terms of vertical stress influence factors given respectively by $I\left(r / r_{0}, z / r_{0}\right)$ and $I(r, z)$ and presented in Tables 2 and 3 . The variation of the vertical stress influence coefficient (factor) at any point $r$ from the centre of the circular foundation and depth $z$ from the ground for uniformly loaded circular foundation is shown in Table 2. Table 3 shows the variation of the vertical stress influence factor (coefficients) for points at any depth $z$ under the centre of a uniformly loaded circular foundation.

Similarly, the vertical displacement field for uniformly distributed load over circular foundation areas on elastic half-space is obtained as Equation (162) by using the point load solving as the Green function. The displacement 
$w(0,0, z)$ is obtained as Equation (165) or alternatively as Equation (166). The displacement $w(r, z=0)$ is obtained as Equation (168) while the displacement $w(0,0,0)$ is obtained as Equation (173) for circular areas under uniform load.

For uniformly distributed line load of finite length the point load solution was used as Green function to obtain the vertical stress field as the integral over the line given by Equation (174) or alternatively as Equation (175). Evaluation of the integral in Equation (175) using the method of transformation of variables and trigonometric substitution gave the vertical stress field in the elastic half-space as Equation (180). The vertical stress field is presented in terms of dimensionless influence coefficients as Equations (181) or (185). The equation for dimensionless influence coefficients for uniformly distributed line load of finite length is Equation (186). Similarly point load solutions for vertical stress field were used as Green function to express the vertical stress field under the centre of a rectangular area under distributed load as the double integrals given by Equation (187). The integral is expressed for uniformly distributed load as Equation (191) and in terms of vertical stress influence factors as Equation (192) where the vertical stress influence factor for vertical stress fields under the centre of uniformly loaded rectangular areas is given by Equation (193). The integration problem is presented in terms of the dimensions of the rectangular area and the depth as Equation (194). By the principle of superposition, the vertical stress influence coefficient for the corner of a rectangular area under uniformly distributed load is found in terms of the dimensions $L, B$ and the depth as Equation (196). The vertical stress influence coefficients are found in terms of non-dimensional factors $m_{1}, n_{1}$ as Equation (201), or alternatively as Equation (205) and Equation (206) for small values of the non-dimensional factors $m_{2}$ and $n_{2}$ and presented in Table 4 . The vertical stress influence factors for the corner parts of rectangular areas under uniform load on elastic foundation is symmetrical with respect to the non-dimensional factors $m_{2}, n_{2}$.

The solutions obtained in this study for point load, uniformly distributed line load of finite length, and uniformly distributed load over circular and rectangular areas agree with results from the technical literature. The results presented in Table 2 for the vertical stress fields in elastic half-space due to uniformly distributed load on a circular area are identical with the previous study presented by Ike $[22,23,39,40]$. The results presented in Table 1 for vertical stress field influence coefficient due to point load at the origin of an elastic half-space and Table 3 for vertical stress influence coefficients under the centre of a uniformly loaded circular area on a half-space are identical with previous results obtained by Ike $[23,39,40]$. Similarly, the results presented in Table 4 for the vertical stress influence coefficient for the corner points of rectangular area on an elastic half-space are in agreement with results obtained by Ike [23] and by Onah et al [32].

\section{Conclusions}

The conclusions of the present work are as follows:

The Trefftz displacement potential function method has been successfully used to find stress fields, and displacement fields in an elastic half-space $(-\infty \leq x \leq \infty,-\infty \leq y \leq \infty, 0 \leq z \leq \infty)$ due to point load acting at the origin $(0,0,0)$, uniformly distributed line load of finite length, and uniformly distributed loads over circular areas and over rectangular areas on the half-space.

It was proved that the Trefftz displacement potential functions are solutions to the Navier-Lamé displacement formulation of elastostatic problems of the elastic half-space, thus the Trefftz functions satisfying the fundamental equations of 3D elasticity theory namely kinematic, generalised Hooke's law and the differential equations of equilibrium.

Equilibrating strain, stress and displacement fields were derived in terms of the Trefftz displacement potential functions.

The Trefftz displacement potential functions method simplifies the 3D elasticity problem of the half-space from a problem of solving a system of fifteen equations to one of finding a scalar potential function of the Cartesian coordinate variables that satisfy the boundary conditions imposed by the loads.

The Trefftz displacement potential functions are derived from the application of the exponential Fourier transform technique to the governing Laplace PDE which the Trefftz function must satisfy to qualify as solutions of the Navier-Lamé displacement equations of equilibrium.

The application of the exponential Fourier transformation on the governing Laplace PDE reduced the problem further from a PDE to a homogeneous ODE which was solved to obtain the solution in exponential Fourier transform space in terms of two integration constants.

Enforcement of the boundedness requirement simplified the solution to an exponential function with one unknown integration constant (in the exponential Fourier transform space).

The unknown constant of integration was obtained using the boundary condition requirement of equilibrium of internal vertical stress resultant and the applied vertical point load.

The Trefftz displacement potential function obtained for point load at the origin of the half-space is a logarithmic potential function that is singular and undefined at the origin, the point of application of the point load. This results in vertical stress fields that are singular and undefined at the origin for the case of point load at the origin.

Point load solutions for vertical stress fields were used as Green functions to determine the vertical stress fields due to distributed loads on lines of finite length and on circular and rectangular areas; thus, expressing the problems as integration problems over lines, and the circular and 
rectangular areas of the load.

Results for vertical stress fields are presented in terms of non-dimensional vertical stress influence factors (coefficients), which are evaluated and tabulated.

The results of stress and displacement fields for the various load cases obtained by the Trefftz displacement potential function method agrees with results obtained by other researchers who used other methods including stress harmonic functions and Bessel functions.

\section{Acknowledgments}

The authors are grateful to the Editor-in-Chief and all members of the Review and Editorial Boards of this journal for their positive and constructive roles in the activities that contribute to the publication of this paper.

\section{Funding}

The research was funded by the authors.

\section{REFERENCES}

[1] Apostol B.F., "Elastic equilibrium of the Half-Space revisited, Mindlin and Boussinesq problems," Journal of Elasticity, vol. 125, pp. $139-148,2016$. DOI: 10.1007/s10659-016-9574-5.

[2] Selvadurai A.P.S., "On Frohlich's solution for Boussinesq problem," International Journal for Numerical and Analytical Methods in Geomechanics, vol.38, pp.925 - 934, 2014.

[3] Kachanov M.L., Shefiro B., Tsukrov I., "Handbook of Elasticity Solutions," Springer Science and Business Media Kluwer Academic Publishers, Dordrencht, The Netherlands, 2003.

[4] Palaniappan, D., "A general solution of equations of equilibrium in linear elasticity," Applied Mathematical Modelling, vol.35, pp. 5494-5499, 2011.

[5] Barber J.R., "Elasticity," $3^{\text {rd }}$ Revised Edition, Springer Science and Business Media, Dordrencht, The Netherlands, 2010 .

[6] Sadd M.H., "Elasticity Theory Applications and Numerics" Elsevier Butterworth Heinemann Amsterdam, 2009.

[7] Selvadurai A.P.S., “On Boussinesq'sproblem," Internation al Journal of Engineering Science, Pergamon, vol. 39, pp. 317-322, 2001. www.elsevier.com/locate/ijengsci.

[8] Zhou S., Gao X.L., "Solutions of half-space and half-plane contact problems based on surface elasticity", ZeitschriftfürangewandleMathematik und Physik (ZAMP), vol. 64, no.1, pp.145 - 166, 2013. DOI: 10.1007/500088-012-0205-0.

[9] Hazel A., "MATH 350211 Elasticity,"www.maths.manches ter.ac.uk/ ahazel/MATHS. Nov. 30, 2015.

[10] Abeyartne R., (2012). "Continuum Mechanics Volume II of Lecture notes on the mechanics of elastic solids," Cambridge, $\mathrm{http} / /$ web.mit.edu/abeyartne/lecture_notes html $11^{\text {th }}$ May, 2012. Retrieved Jan. 2019.

[11] Padio-Guidugli P., Favata, A., "Elasticity for Geotechnicians: A modern exposition of Kelvin, Boussinesq, Flammant, Cerrutti, Melan and Mindlin problems," Solid Mechanics and its Applications, Springer, New York, 2014.

[12] Davis R.O., Selvadurai A.P.S., "Elasticity and Geomechanics," Cambridge University Press, Cambridge, 1996.

[13] Sitharam, T.G., Govinda - Reju L., "Applied Elasticity for Engineers, Module, Elastic Solutions and Applications in Geomechanics," Available online at 14.139.172.204/nptel/1/ CSE/web/105108070/module8/lecture 17.pdf. Retrieved Feb. 2018.

[14]Zhou S., Gao X.L., "Solutions of a generalized half-plane and half-space Cerrutti problems with surface effects," Zeitschrift fur Angewandle Mathematik und Physik,(ZAMP), vol.66,pp.1125 - 1142, 2014.

[15] Favata A., “On the Kelvin problem,"https/arxiv.org/pdf/120 2.1719. 2012. Accessed 10/11/2018.

[16] Ike C.C., "Fourier sine transform method for solving the Cerrutti problem of elastic half-plane in plane strain," Mathematical Modelling in Civil Engineering, vol.14, no.1, pp. 1 - 11, 2018. DOI: 10.2478/mmce_2018-0001.

[17] Ike C.C., Mama B.O., Onah H.N.,Nwoji C.U.,"Trefftz harmonic function method for solving Boussinesq problem," Electronic Journal of Geotechnical Engineering, Vol. (22.12) pp. 4589 - 4601,2017. www.ejge.com.

[18] Nwoji C.U., Onah H.N., Mama B.O., Ike C.C., "Solution of Elastic half space problem using Boussinesq displacement potential functions," Asian Journal of Applied Sciences, vol.05, no.05, pp. 1087-1093, 2017.

[19] Ike C.C., Onah H.N., Nwoji, C.U., "Bessel functions for axisymmetric elasticity problems of the elastic half space soil, a potential function method," Nigerian Journal of Technology, vol.36, no.3, pp.773 - 781, 2017. http//dx.doi.org/10.4314/nijt.v36i3.16.

[20] Nwoji C.U., Onah H.N., Mama B.O., Ike C.C., "Solution of the Boussinesq problem of half-space using Green and Zerna displacement potential function method," Electronic Journal of Geotechnical Engineering, vol. (22.10), pp. 4305 - 4314, 2017. www.ejge.com.

[21] Ike C.C., "General solutions for axisymmetric elasticity problems of elasticity half space using Hankel transform method," International Journal of Engineering and Technology, vol.10, no.2, pp.565-580, April-May 2018. DOI: $10.21817 / \mathrm{ijet} / 2018 / \mathrm{v} 10 \mathrm{i} 2 / 181002112$.

[22] Ike C.C., (2018). "Hankel transform method for solving axisymmetric elasticity problems of circular foundation on semi-infinite soils," International Journal of Engineering and Technology, vol.10, no.2, pp. 549-564, April-May 2018.DOI: $10.21817 / \mathrm{ijet} / 2018 / \mathrm{v} 10 \mathrm{i} 2 / 181002111$.

[23] Ike C.C., "Principles of Soil Mechanics," De-Adroit Innovation, Enugu, 2006. 
[24] Boussinesq J., "Application des potentials al'etude de l'equilibre et des mouvements des solideselastiques," Paris, Ganther - Villars, 1885.

[25] Trefftz E., "MathematischeElastizitatetheorie," in Handbuch der Physik Ed VI, Berlin Springer, 1928.

[26] Green A.E., Zerna W., "Theoretical Elasticity," Oxford University Press, London, 1954.

[27] Love A.E.H., "Mathematical Theory of Elasticity," $4^{\text {th }}$ Edition, Dover Publications Inc., New York, 1944.

[28] Chau K.T., "Analytic Methods in Geomechanics," CRC Press, Taylor and Francis Group, New York, 2012. ISBN: 10:1466555858.

[29] Ike C.C., "First principles derivation of a stress function for axially symmetric elasticity problems, and application to Boussinesq problem," Nigerian Journal of Technology, vol.36, no.3, pp. 767-772, 2017.http://dx.doi.or $\mathrm{g} / 10.4314 /$ nijt/v36i3.15.

[30] Egorov Y.E., Screbrjanyi R.V., "Determination of stresses in a rigid circular foundation," $2^{\text {nd }}$ Asian Regional Conference on Soil Mechanics and Foundation Engineering, Tokyo Japan, vol.1, pp. $246-250,1963$.

[31] Harr M.E., "Foundations of Theoretical Soil Mechanics," McGraw Hill, New York, 1966.

[32] Onah H.N., Mama B.O., N Nwoji C.U., Ike C.C., "Boussinesq displacement potential functions method for finding vertical stresses and displacement fields due to distributed load on elastic half space," Electronic Journal of Geotechnical Engineering, vol. (22.15), pp.5687 - 5709, 2017. www.ejge.com.

[33] Ike C.C., "Elzaki transform method for finding solutions to two-dimensional elasticity problems in polar coordinates formulated using Airy stress functions," Journal of Computational Applied Mechanics, vol. 51, issue 2, pp.302-310, 2020. DOI: 10.22059/jcamech.2020.296012.47 2.

[34] Onah H.N., Ike C.C., Nwoji C.U., Mama B.O., "Theory of elasticity solution for stress fields in semi-infinite linear elastic soil due to distributed load on the boundary using the Fourier transform method," Electronic Journal of Geotechnical Engineering, vol. (22.13), pp. 4945 - 4962, 2017. www.ejge.com.
[35] Ike C.C., "Exponential Fourier integral transform method for the stress analysis of boundary load on soil," Mathematical Modelling of Engineering Problems, vol. 5, no 1, pp 33 - 39, 2018. http//doi.org/10.18280/mmep.050105.

[36] Ike C.C., "Solution of elasticity problems in two-dimensional polar coordinates using Mellin transform," Journal of Computational Applied Mechanics, vol. 50, issue 1, pp 174 181, 2019. DOI: 10.22059/jcamech.2019.278288.370

[37] Ike C.C., (2018). “On Maxwell's stress functions for solving three dimensional elasticity problems in the theory of elasticity," Journal of Computational Applied Mechanics, vol. 49, issue 2, pp 342 - 350, 2018. DOI: 10.22059/jcamech.2018.266787.330.

[38] Ike C.C., "Hankel transformation method for solving the Westergaard problem for point, line and distributed loads on elastic half-space," Latin American Journal of Solids and Structures, vol. 16, no 1, pp 1 - 19, 2019. http://dx.doi.org/10.1590/1679-78255313.

[39] Ike C.C., "Fourier-Bessel transform method for finding vertical stress fields in axisymmetric elasticity problems of elastic half-space involving circular foundation areas," Advances in Modelling and Analysis A, vol. 55, no 4, pp 207 -216, 2018. https://doi.org/10.18280/ama_a.550405.

[40] Ike C.C., "Love stress function method for solving axisymmetric elasticity problems of the elastic half-space," Electronic Journal of Geotechnical Engineering, vol. (24.3), pp. $663-706,2019$. www.ejge.com.

[41] Ike C.C., "Fourier cosine transform method for solving the elasticity problem of point load on an elastic half-plane," International Journal of Scientific and Technology Research, vol. 9, issue 4, pp. 1850 - 1856, April 2020.

[42] Ike C.C., Onah H.N., Onyia M.E., Mama B.O., Nwoji C.U., (2020). "First principles derivation of displacement and stress functions for three dimensional elasticity problems and application to the flexural analysis of thick circular plates," Journal of Computational Applied Mechanics, vol. 51, issue 1, pp. 184 - 198, 2020. DOI:10.22059/jcamech.2020.29598 9.471 .

[43] Ike C.C., "Cosine integral transformation method for solving the Westergaard problem in elasticity of the half-space," Civil Engineering Infrastructures Journal, vol. 53, issue 2, pp.313-339, 2020. DOI: 10.22059/ceij.2020.285125.1596. 